\title{
Spatial structure of anomalously localized states in disordered conductors.
}

\author{
Alexander D. Mirlin \\ Institut für Theorie der Kondensierten Materie, Universität Karlsruhe, 76128 Karlsruhe, \\ Germany \\ and Petersburg Nuclear Physics Institute, 188350 Gatchina, St.Petersburg, Russia.
}

(April 16, 2021)

\begin{abstract}
The spatial structure of wave functions of anomalously localized states (ALS) in disordered conductors is studied in the framework of the $\sigma$-model approach. These states are responsible for slowly decaying tails of various distribution functions. In the quasi-one-dimensional case, properties of ALS governing the asymptotic form of the distribution of eigenfunction amplitudes are investigated with the use of the transfer matrix method, which yields an exact solution to the problem. Comparison of the results with those obtained in the saddle-point approximation to the problem shows that the saddle-point configuration correctly describes the smoothed intensity of an ALS. On this basis, the properties of ALS in higher spatial dimensions are considered. We study also the ALS responsible for the asymptotic behavior of distribution functions of other quantities, such as relaxation time, local and global density of state. It is found that the structure of an ALS may be different, depending on the specific quantity, for which it constitutes an optimal fluctuation. Relations between various procedures of selection of ALS, and between asymptotics of corresponding distribution functions, are discussed.
\end{abstract}

PACS numbers: 71.55.Jv, 71.20.-b, 05.40.+j 


\section{INTRODUCTION}

Mesoscopic fluctuations of various physical quantities in disordered systems have been intensively investigated during the last decade [1]. These fluctuations originating from the quantum coherence of wave functions are typically much stronger than what usual statistical considerations would predict. In particular, it was found by Altshuler, Kravtsov and Lerner [2] that distribution functions of conductance, density of states (DOS), local density of states (LDOS), and relaxation times have slowly decaying logarythmically-normal $(\mathrm{LN})$ asymptotics at large values of the arguments. These results were obtained within the renormalization group (RG) treatment of the field-theoretical $\sigma$-model describing the lowmomenta physics of the problem. The validity of this RG approach is restricted to $2 \mathrm{D}$ and $2+\epsilon$-dimensional systems, with $\epsilon \ll 1$.

On the other hand, the conductance, LDOS and relaxation times fluctuations in strictly 1D disordered chains, where all states are strongly localized, were studied with the use of Berezinski and Abrikosov-Ryzhkin techniques [3 5]. The corresponding distributions were found to be of the LN form, too. It was conjectured on the basis of this similarity [2, 4, 6] that even in a metallic sample there is a finite probability to find "almost localized" eigenstates. Precise meaning of these words remained however obscure.

More recently, the interest to statistical properties of eigenfunctions in disordered and chaotic systems started to grow. On the experimental side, it was motivated by the possibilty of fabrication of small systems (quantum dots) with well resolved electron energy levels [7]. Conductance fluctuations of such a dot are related to statistical properties of wave function amplitudes [8]. Besides, the microwave cavity technique [9] allows to observe experimentally spatial fluctuations of electromagnetic wave amplitude in chaotis or disordered cavities [10].

On the theoretical side, the recent progress is due to the observation [11] that the statistics of eigenfunctions intensities can be very efficiently studied with making use of the supersymmetry technique. This allows one to reformulate the problem in terms of the supermatrix $\sigma$-model [12]. It was found that the zero-dimensional approximation to this $\sigma$-model reproduces the Random Matrix Theory (RMT) results. In the case of quasi-1D geometry, the model was solved exactly by means of the transfer-matrix method [17], which allowed us to calculate various statistical properties of eigenfunctions 11, 17, 20. It was found that the distribution function $\mathcal{P}(u)$ of eigenfunction intensities $u=\left|\psi^{2}(\boldsymbol{r})\right|$ deviates slightly from its RMT form for not too large $u$, but decreases much slower than RMT predicts, in its far "tail". In $d>1$ dimensions, the results for $\mathcal{P}(u)$ were obtained by means of perturbative method [20] and saddle-point approximation [21], and are qualitatively similar to the quasi-1D behavior.

It should be noted that the saddle-point method for the supermatrix $\sigma$-model used in Ref. [21] and suggested previously by Muzykantskii and Khmelnitskii [22 turned out to be a powerful tool to study the asymptotic "tails" of various distributions. In particular, it was used in [22,23] to study the long-time relaxation phenomena, and in 24 to find the asymptotical behavior of the distribution of LDOS. The obtained decrease rates are much slower than those given by the perturbation theory. In $2 \mathrm{D}$, the far asymptotics are of LN type [23,24], in agreement with the RG results of [2]. The asymptotic behavior of the distributions was again attributed to the effect of almost localized states [22, 20,21. Moreover, it was conjectured in [22,21] that the form of the saddle-point solution of the 
$\sigma$-model directly describes the electron density of such a state.

The purpose of this paper is to study in most detail the spatial structure of the anomalously localized states (ALS). The paper is organized as follows. In section II we review briefly the previously obtained results for the distribution of eigenfunctions intensity. In section III, which is the central one for the paper, we study the case of a quasi-1D geometry of the sample. We present the exact calculation of the properties of ALS responsible for the asymptotics of $\mathcal{P}(u)$. In subsection $\| I I \mathrm{~A} 1$ the average intensity $\left\langle\left|\psi^{2}(\boldsymbol{r})\right|\right\rangle$ of such a state is found for a sample with the length $L \gg \xi$, where $\xi$ is the localization length. The state with an anomalously high local intensity $\left|\psi^{2}(0)\right|=u$ is characterized by an effective localization length $\xi_{e f} \sim(\xi / u S)^{1 / 2}$ (here $S$ is the wire cross-section), where most of its normalization is concentrated. Outside this central region, $\left\langle\left|\psi^{2}(r)\right|\right\rangle$ decreases as $1 / r^{2}$. Finally, in the vicinity of the observation point there is a sharp change of $\left\langle\left|\psi^{2}\right|\right\rangle$ from $\left|\psi^{2}(0)\right|=u$ to $\left\langle\left|\psi^{2}\right|\right\rangle=(u / \xi S)^{1 / 2} / 2$. In subsection III A2 we repeat this calculation in the case of metallic sample with $L \ll \xi$. We find that ALS have exactly the same form of $\left\langle\left|\psi^{2}(r)\right|\right\rangle$, if $u \gg \xi / S L^{2}$. The latter condition means that $\xi_{e f} \ll L$, i.e. the state plays a role of a localized one even from the point of view of a short sample with the length $L$.

In subsection $\amalg$ II B we study the fluctuations of intensity $\left|\psi^{2}(r)\right|$ of an ALS. We find that these fluctuations are of the RMT form. The narrow region around the observation point where the quasi-jump of $\left\langle\left|\psi^{2}\right|\right\rangle$ occurs, is an exception; here the fluctuations are suppressed.

Comparing our results with the corresponding saddle-point solution [21], we conclude that the latter describes exactly the form of the average density $\left\langle\left|\psi^{2}(r)\right|\right\rangle$, up to a normalization factor and without the quasi-jump around the observation point $r=0$. This allows us to generalize the results to the spatial dimension $d>1$ (section $\mathbb{E}$ ). In section $\nabla$ we discuss and compare the spatial structure of ALS corresponding to asymptotical behavior of various distribution functions. In Sec.VI we discuss briefly some special features of the ALS located close to the sample edge, and dependence of the results on the symmetry of the ensemble. Our results are summarized in section $\nabla 11$.

\section{DISTRIBUTION OF EIGENFUNCTION AMPLITUDES: OVERVIEW OF RESULTS.}

In this section, we have collected some results for the eigenfunction intensity distribution function obtained with use of the supermatrix $\sigma$-model formalism. The $\sigma$-model action reads

$$
S[Q]=-\frac{\beta}{2} \int d^{d} r \operatorname{Str}\left[\frac{\pi \nu_{0} D}{4}(\nabla Q)^{2}-\pi \nu_{0} \eta \Lambda Q\right]
$$

Here $Q(\boldsymbol{r})$ is a $4 \times 4$ or $8 \times 8$ supermatrix field for the cases of unitary symmetry (broken time reversal invariance) and orthogonal symmetry (unbroken time reversal invariance) respectively. We will label the formulas for the first case by the index $U$, and for the second case by the index $O$. The matrix $\Lambda$ is defined as $\Lambda=\operatorname{diag}\{1,1,-1,-1\}$ (U) or $\Lambda=\operatorname{diag}\{1,1,1,1,-1,-1,-1,-1\}(\mathrm{O})$ and the coefficient $\beta$ is equal to $\beta=2(\mathrm{U})$ or $\beta=1$

(O). Furthermore, Str stands for the supertrace (trace over bosonic components minus trace over fermionic ones), $D$ is the diffusion constant, $\eta$ is the level broadening (or, else, $(-2 i) \times$ 
frequency) and $\nu_{0}$ is the mean DOS. We do not go into details of the supersymmetric formalism here, which can be found e.g. in [25,26, 19]. Let us define now the function $Y\left(Q_{0}\right)$ as

$$
Y\left(Q_{0}\right)=\int_{Q\left(\boldsymbol{r}_{\mathbf{0}}\right)=Q_{0}} D Q(r) \exp \{-S[Q]\}
$$

For the invariance reasons, the function $Y\left(Q_{0}\right)$ turns out to be dependent in the unitary symmetry case on the two scalar variables $1 \leq \lambda_{1}<\infty$ and $-1 \leq \lambda_{2} \leq 1$ only, which are eigenvalues of the "retarded-retarded" block of the matrix $Q_{0}$. Moreover, in the limit $\eta \rightarrow 0$ (at a fixed value of the system volume) only the dependence on $\lambda_{1}$ persists:

$$
Y\left(Q_{0}\right) \equiv Y\left(\lambda_{1}, \lambda_{2}\right) \rightarrow Y_{a}\left(2 \pi \nu_{0} \eta \lambda_{1}\right)
$$

The distribution $\mathcal{P}(u)$ of the eigenfunctions intensities $u=\mid \psi\left(\left.\boldsymbol{r}_{\mathbf{0}}\right|^{2}\right.$ is then given by 11, 15]

$$
\mathcal{P}(u)=\frac{1}{V} \frac{d^{2}}{d u^{2}} Y_{a}(u)=\left.\frac{1}{V} \frac{d^{2}}{d u^{2}} Y\left(\lambda_{1}=\frac{u}{2 \pi \nu_{0} \eta}\right)\right|_{\eta \rightarrow 0}
$$

In the case of orthogonal symmetry, $Y\left(Q_{0}\right) \equiv Y\left(\lambda_{1}, \lambda_{2}, \lambda\right)$, where $1 \leq \lambda_{1}, \lambda_{2}<\infty$ and $-1 \leq \lambda \leq 1$. In the limit $\eta \rightarrow 0$, the relevant region of values is $\lambda_{1} \gg \lambda_{2}, \lambda$, where

$$
Y\left(Q_{0}\right) \rightarrow Y_{a}\left(\pi \nu_{0} \eta \lambda_{1}\right)
$$

The distribution of eigenfunction intensities is in this case expressed through the function $Y_{a}$ as follows [1]:

$$
\begin{aligned}
\mathcal{P}(u) & =\frac{1}{\pi V u^{1 / 2}} \int_{u / 2}^{\infty} d z(2 z-u)^{-1 / 2} \frac{d^{2}}{d z^{2}} Y_{a}(z) \\
& =\frac{2 \sqrt{2}}{\pi V u^{1 / 2}} \frac{d^{2}}{d u^{2}} \int_{0}^{\infty} \frac{d z}{z^{1 / 2}} Y_{a}(z+u / 2)
\end{aligned}
$$

In the diffusive sample, typical configurations of the $Q$-field are nearly constant in space, so that one can approximate the functional integral (2.2) by an integral over a single supermatrix $Q$. This procedure, which makes the problem effectively zero-dimensional and is known as zero-mode approximation, gives

$$
Y_{a}(z) \approx e^{-V z} \quad(O, U)
$$

and consequently,

$$
\begin{array}{cc}
\mathcal{P}(u) \approx V e^{-u V} & (U), \\
\mathcal{P}(u) \approx \sqrt{\frac{V}{2 \pi u}} e^{-u V / 2} & (O),
\end{array}
$$

which are just the RMT results for the Gaussian Unitary Ensemble (GUE) and Gaussian Orthogonal Ensemble (GOE) respectively [27].

For the case of quasi-1D geometry one can solve the problem exactly, by evaluating the integral in (2.2) with making use of the transfer-matrix method [1]. As a result, one gets an expression for $\mathcal{P}(u)$ depending on a scaling parameter $x=L / \xi$, where $L$ is the 
sample length, $\xi=2 \pi \nu_{0} S D$ is the localization length, and $S$ is the transverse cross-section 11, 19, 20. In particular, in the case of a short (metallic) sample, $x \ll 1$, we find for the distribution of normalized intensity $y=u V$ :

$$
\begin{array}{rlrl}
\mathcal{P}^{(U)}(y) & =e^{-y}\left[1+\frac{\alpha x}{6}\left(2-4 y+y^{2}\right)+\ldots\right] ; & & y \lesssim x^{-1 / 2} \\
\mathcal{P}^{(O)}(y)= & \frac{e^{-y / 2}}{\sqrt{2 \pi y}}\left[1+\frac{\alpha x}{6}\left(\frac{3}{2}-3 y+\frac{y^{2}}{2}\right)+\ldots\right] ; & & y \lesssim x^{-1 / 2} \\
\mathcal{P}(y) & \sim \exp \left\{\frac{\beta}{2}\left[-y+\frac{\alpha}{6} y^{2} x+\ldots\right]\right\} ; & & x-1 / 2 \lesssim y \lesssim x^{-1} \\
\mathcal{P}(y) \sim \exp [-2 \beta \sqrt{y / x}] ; & & y \gtrsim x^{-1}
\end{array}
$$

Here the coeficient $\alpha$ is equal to $\alpha=2\left[1-3 L_{-} L_{+} / L^{2}\right]$, where $L$ is the sample length and $L_{-}$, $L_{+}$are the distances from the observation point $\boldsymbol{r}_{\mathbf{0}}$ to the sample edges. If one is interested in the distribution function $\mathcal{P}(y)$ averaged over the position of the point $\boldsymbol{r}_{\mathbf{0}}$ within the sample, one has to replace $\alpha$ in eqs.(2.10), (2.11) by its average value equal to unity, and in eq.(2.12) by its maximum value equal to 2. Such averaged distribution functions were considered in our previous publications, Ref. [11.19,.20]. It is straightforward, however, to generalize the derivation and to get the formulas (2.10) $-(2.12)$ for the position-dependent distribution functions. Note that $g=1 / x$ is for small $x$ just the dimensionless conductance of the sample: $g=2 \pi \nu_{0} D S / L=G /\left(e^{2} / h\right)$. We see, that for not too large amplitudes $y$, Eqs. 2.10), (2.11) are valid which are just the RMT results with relatively small corrections. In the intermediate region (2.12), the correction in the exponent is small compared to the leading term but much larger than unity, so that $\mathcal{P}(y) \gg \mathcal{P}_{R M T}(y)$ though $\ln \mathcal{P}(y) \simeq$ $\ln \mathcal{P}_{R M T}(y)$. Finally, in the large amplitude region, (2.13), the distribution function $\mathcal{P}(y)$ differs completely from the RMT prediction. We will present eq.(2.13) in a more precise form in Sec. III. Note that it is not valid when the observation point is located close to the sample boundary, as will be explained in Sec.VIA. The asymptotic behavior (2.13) is the same as in the case of insulating sample, $L \gg \xi$, when the distribution $\mathcal{P}(u)$ takes the form

$$
\begin{aligned}
& \mathcal{P}^{(U)}(u) \simeq \frac{8 \xi^{2} S}{L}\left[K_{1}^{2}(2 \sqrt{u S \xi})+K_{0}^{2}(2 \sqrt{u S \xi})\right] \\
& \mathcal{P}^{(O)}(u) \simeq \frac{2 \xi^{2} S}{L} \frac{K_{1}(2 \sqrt{u S \xi})}{\sqrt{u S \xi}}
\end{aligned}
$$

This allowed us to conjecture in Ref. [20] that the asymptotic behavior (2.13) is controlled by the probability to have a quasilocalized eigenstate with an effective spatial extent much less than $\xi$. We will prove this rigorously in the present paper.

In $d>1$ dimensions the problem can not be solved exactly anymore, and some approximate methods of evaluation of the integral (2.2) are necessary. For moderately large amplitudes $y \lesssim \kappa^{-1 / 2}$, a perturbative treatment of non-zero modes of the $\sigma$-model in the weak localization region is possible [20]. Here $\kappa$ is the usual parameter of the perturbation theory [2] defined as

$$
\kappa=\sum_{\boldsymbol{q}} \frac{1}{2 \pi \nu_{0} D \boldsymbol{q}^{2} V} ;
$$

the summation goes over the eigenmodes of the Laplace operator in the sample. The result for $\mathcal{P}(y)$ is: 


$$
\begin{aligned}
& \mathcal{P}^{(U)}(y)=e^{-y}\left[1+\kappa\left(2-4 y+y^{2}\right)+\ldots\right] \\
& \mathcal{P}^{(O)}(y)=\frac{e^{-y / 2}}{\sqrt{2 \pi y}}\left[1+\kappa\left(\frac{3}{2}-3 y+\frac{y^{2}}{2}\right)+\ldots\right]
\end{aligned}
$$

In particular, in quasi-1D systems $\kappa=1 / 6 g$, and Eqs.(2.10), (2.11) are reproduced. For $d \geq 2$ the corresponding sum over momenta $\boldsymbol{q}$ diverges at large $|\boldsymbol{q}|$ and is to be cut off at $|\boldsymbol{q}| \sim l^{-1}$. This gives $\kappa=\frac{1}{4 \pi^{2} \nu_{0} D} \ln (L / l)$ for $d=2$ and $\kappa \sim\left(k_{F} l\right)^{2}$ for $d=3$, where $k_{F}$ is the Fermi momentum.

In the region of large amplitudes $y \gtrsim \kappa^{-1 / 2}$, Eq.(2.17), (2.18) loose their validity. In this region, the distribution $\mathcal{P}(y)$ can be found [21] by using the saddle-point approximation. For $d=2,3$ the result is

$$
\begin{aligned}
& \mathcal{P}(y) \sim \exp \left\{\frac{\beta}{2}\left(-y+\kappa y^{2}+\ldots\right)\right\}, \quad \kappa^{-1 / 2} \lesssim y \lesssim \kappa^{-1} \\
& \mathcal{P}(y) \sim \exp \left\{-\frac{\beta}{8 \kappa} \ln ^{d}(\kappa y)\right\}, \quad y \gtrsim \kappa^{-1}
\end{aligned}
$$

In the next two sections, we will study in detail the structure of the states, which are responsible for the far asymptotics (2.13), (2.20). For definitness, we will consider the unitary symmetry case; generalization to the orthogonal ensemble is completely straightforward, and we will just quote the (minor) changes in the results in Sec.VIB.

\section{ANOMALOUSLY LOCALIZED STATES IN QUASI-1D SAMPLES}

\section{A. Average form of the eigenfunction corresponding to an anomalously high local amplitude.}

In this section, we study the average intensity $\left\langle\left|\psi^{2}(\boldsymbol{r})\right|\right\rangle_{u}$ of a wave function with the condition that $\left|\psi^{2}(0)\right|=u$. For convenience, we have put the observation point in the coordinate origin; the sample edges are at $r=-L_{-}$and $r=L_{+}$; the sample length is $L=L_{-}+L_{+}$. Formally, $\left\langle\left|\psi^{2}(\boldsymbol{r})\right|\right\rangle_{u}$ is defined as

$$
\left\langle\left|\psi^{2}(\boldsymbol{r})\right|\right\rangle_{u}=\frac{\mathcal{Q}(u, \boldsymbol{r})}{\mathcal{P}(u)},
$$

where

$$
\begin{aligned}
\mathcal{P}(u) & =\frac{1}{\nu_{0} V}\left\langle\sum_{\alpha} \delta\left(\left|\psi_{\alpha}(0)\right|^{2}-u\right) \delta\left(E-E_{\alpha}\right)\right\rangle \\
\mathcal{Q}(u, \boldsymbol{r}) & =\frac{1}{\nu_{0} V}\left\langle\sum_{\alpha}\left|\psi_{\alpha}(\boldsymbol{r})\right|^{2} \delta\left(\left|\psi_{\alpha}(0)\right|^{2}-u\right) \delta\left(E-E_{\alpha}\right)\right\rangle,
\end{aligned}
$$

where $\psi_{\alpha}$ are eigenfunctions of the Hamiltonian, and $E_{\alpha}$ are the corresponding eigenvalues. The here defined function $\mathcal{P}(u)$ is just the distribution function of local intensity discussed in the preceding section. As has been mentioned above, it was calculated in the quasi-1D case, with making use of the $\sigma$-model representation, eqs.(2.4), (2.6), and the transfer-matrix method [11,[19]. The result reads 


$$
\mathcal{P}(u)=\frac{1}{V} \frac{\partial^{2}}{\partial u^{2}}\left\{W^{(1)}\left(u S \xi, \tau_{+}\right) W^{(1)}\left(u S \xi, \tau_{-}\right)\right\}
$$

where the function $W^{(1)}(z, \tau)$ satisfies the equation

$$
\frac{\partial W^{(1)}(z, \tau)}{\partial \tau}=\left(z^{2} \frac{\partial^{2}}{\partial z^{2}}-z\right) W^{(1)}(z, \tau)
$$

and the boundary condition

$$
W^{(1)}(z, 0)=1
$$

The solution to eqs.(3.5), (3.6) can be found in terms of the Lebedev-Kontorovich expansion

$$
W^{(1)}(z, \tau)=2 z^{1 / 2}\left\{K_{1}\left(2 z^{1 / 2}\right)+\frac{2}{\pi} \int_{0}^{\infty} d \nu \frac{\nu}{1+\nu^{2}} \sinh \frac{\pi \nu}{2} K_{i \nu}\left(2 z^{1 / 2}\right) e^{-\frac{1+\nu^{2}}{4} \tau}\right\}
$$

Now we turn to evaluation of the function $\mathcal{Q}(u, r)$ defined in eq.(3.3). Detailed exposition of the method used can be found in Ref. [19], and we will mainly follow the notations of this paper. We start from expressing the moments $\left\langle|\psi(\boldsymbol{r})|^{2}|\psi(0)|^{2 q}\right\rangle$ in terms of the Green's functions

$$
\begin{aligned}
& \left\langle|\psi(\boldsymbol{r})|^{2}|\psi(0)|^{2 q}\right\rangle \\
& \equiv \frac{1}{\nu_{0} V}\left\langle\sum_{\alpha}\left|\psi_{\alpha}(\boldsymbol{r})\right|^{2}\left|\psi_{\alpha}(0)\right|^{2 q} \delta\left(E-E_{\alpha}\right)\right\rangle \\
& =\lim _{\eta \rightarrow 0} \frac{i^{q-1}}{2 \pi \nu_{0} V}(2 \eta)^{q}\left\langle G_{R}^{q}(0,0) G_{A}(\boldsymbol{r}, \boldsymbol{r})\right\rangle,
\end{aligned}
$$

where

$$
G_{R, A}\left(\boldsymbol{r}_{\mathbf{1}}, \boldsymbol{r}_{\mathbf{2}}\right)=\left\langle\boldsymbol{r}_{\mathbf{1}}\left|(E-\hat{H} \pm i \eta)^{-1}\right| \boldsymbol{r}_{\mathbf{2}}\right\rangle
$$

Here $\hat{H}$ is the Hamiltonian which consists of the free part $\hat{H}_{0}$ and the disorder potential $U(\boldsymbol{r})$ :

$$
\hat{H}=\hat{H}_{0}+U(\boldsymbol{r}) ; \quad \hat{H}_{0}=\frac{1}{2 m} \hat{\boldsymbol{p}}^{2} ;
$$

the latter being defined by the correlator

$$
\left\langle U(\boldsymbol{r}) U\left(\boldsymbol{r}^{\prime}\right)\right\rangle=\frac{1}{2 \pi \nu_{0} \tau_{s}} \delta\left(\boldsymbol{r}-\boldsymbol{r}^{\prime}\right)
$$

Next, we write the product of the Green's functions in terms of the integral over a supervector field $\Phi=\left(S_{1}, S_{2}, \chi_{1}, \chi_{2}\right)$ :

$$
\begin{aligned}
G_{R}^{q}(0,0) G_{A}(\boldsymbol{r}, \boldsymbol{r}) & =\frac{i^{1-q}}{q !} \int D \Phi D \Phi^{\dagger}\left(S_{1}(0) S_{1}^{*}(0)\right)^{q} S_{2}(\boldsymbol{r}) S_{2}^{*}(\boldsymbol{r}) \\
& \times \exp \left\{i \int d \boldsymbol{r}^{\prime} \Phi^{\dagger}\left(\boldsymbol{r}^{\prime}\right) \Lambda^{1 / 2}(E+i \eta \Lambda-\hat{H}) \Lambda^{1 / 2} \Phi\left(\boldsymbol{r}^{\prime}\right)\right\}
\end{aligned}
$$

The following steps are [19]: 
i) averaging over the disorder;

ii) introducing a $4 \times 4$ supermatrix variable $R_{\mu \nu}(\boldsymbol{r})$ having the symmetry of the tensor product $\Phi_{\mu}(\boldsymbol{r}) \Phi_{\nu}^{\dagger}(\boldsymbol{r})$;

iii) integrating out the $\Phi$ fields ;

iy) using the saddle-point approximation which leads to the following equation for $R$ :

$$
\begin{aligned}
& R(\boldsymbol{r})=\frac{1}{2 \pi \nu_{0} \tau_{s}} g(\boldsymbol{r}, \boldsymbol{r}) ; \\
& g\left(\boldsymbol{r}_{\mathbf{1}}, \boldsymbol{r}_{\mathbf{2}}\right)=\left\langle\boldsymbol{r}_{\mathbf{1}}\left|\left(E-\hat{H}_{0}-R\right)^{-1}\right| \boldsymbol{r}_{\mathbf{2}}\right\rangle
\end{aligned}
$$

The relevant set of the solutions (the saddle-point manifold) has the form:

$$
R=\sigma \cdot I-\frac{i}{2 \tau_{s}} Q
$$

where $I$ is the unity matrix, $\sigma$ is certain constant, and $Q$ belongs to the coset space $U(1,1 \mid 2)$ and satisfies the condition $Q^{2}=1$. Finally, we find

$$
\begin{aligned}
\left\langle|\psi(\boldsymbol{r})|^{2}|\psi(0)|^{2 q}\right\rangle & =-\frac{1}{2 V} \lim _{\eta \rightarrow 0}\left(2 \pi \nu_{0} \eta\right)^{q} \int D Q\left[Q_{11, b b}^{q}(0) Q_{22, b b}(\boldsymbol{r})\right. \\
& \left.+q \frac{1}{\left(\pi \nu_{0}\right)^{2}} Q_{11, b b}^{q-1}(0) g_{12, b b}(0, \boldsymbol{r}) g_{21, b b}(\boldsymbol{r}, 0)\right] e^{-S\{Q\}}
\end{aligned}
$$

where $S[Q]$ is the $\sigma$-model action defined in eq.(2.2).

Taking into account that $Q(\boldsymbol{r})$ varies slowly on a scale of the mean free path $l$, we have for $\left|\boldsymbol{r}_{\mathbf{1}}-\boldsymbol{r}_{\mathbf{2}}\right| \ll l$

$$
g\left(\boldsymbol{r}_{\mathbf{1}}, \boldsymbol{r}_{\mathbf{2}}\right) \simeq \operatorname{Re}\left\langle G_{R}\left(\boldsymbol{r}_{\mathbf{1}}-\boldsymbol{r}_{\mathbf{2}}\right)\right\rangle+i Q\left(\boldsymbol{r}_{\mathbf{1}}\right) \operatorname{Im}\left\langle G_{R}\left(\boldsymbol{r}_{\mathbf{1}}-\boldsymbol{r}_{\mathbf{2}}\right)\right\rangle
$$

where

$$
\left\langle G_{R}\left(\boldsymbol{r}_{\mathbf{1}}-\boldsymbol{r}_{\mathbf{2}}\right)\right\rangle=\left\langle\boldsymbol{r}_{\mathbf{1}}\left|\left(E-\hat{H}_{0}-\rho+\frac{i}{2 \tau_{s}}\right)^{-1}\right| \boldsymbol{r}_{\mathbf{2}}\right\rangle
$$

is the average one-particle Green's function. In the opposite limiting case $\left|\boldsymbol{r}_{\mathbf{1}}-\boldsymbol{r}_{\mathbf{2}}\right| \gg l$, the Green's function $g\left(\boldsymbol{r}_{\mathbf{1}}, \boldsymbol{r}_{\mathbf{2}}\right)$ vanishes exponentially. Thus, we get

$$
\begin{aligned}
\left\langle|\psi(\boldsymbol{r})|^{2}|\psi(0)|^{2 q}\right\rangle \simeq & -\frac{1}{2 V} \lim \left(2 \pi \nu_{0} \eta\right)^{q} \int D Q Q_{11, b b}^{q}(0) Q_{22, b b}(\boldsymbol{r}) e^{-S\{Q\}}, \quad r \gg l \\
\left\langle|\psi(\boldsymbol{r})|^{2}|\psi(0)|^{2 q}\right\rangle & \simeq-\frac{1}{2 V} \lim \left(2 \pi \nu_{0} \eta\right)^{q} \int D Q\left[Q_{11, b b}^{q}(0) Q_{22, b b}(0)\right. \\
& \left.+q k_{d}(r) Q_{11, b b}^{q-1}(0) Q_{12, b b}(0) Q_{21, b b}(0)\right] e^{-S\{Q\}}, \quad r \ll l
\end{aligned}
$$

where 


$$
k_{d}(r)=\frac{\left|\operatorname{Im}\left\langle G_{R}(r)\right\rangle\right|^{2}}{\left(\pi \nu_{0}\right)^{2}}
$$

depends on short-scale dimensionality $d$ of the sample [28]. In particular, for a strip $(d=2)$ or a wire $(d=3)$ we have

$$
\begin{aligned}
& k_{2}(r)=J_{0}^{2}\left(k_{F} r\right), \\
& k_{3}(r)=\frac{\sin ^{2}\left(k_{F} r\right)}{\left(k_{F} r\right)^{2}},
\end{aligned}
$$

respectively.

Now we evaluate the $Q$-integrals in eqs.(3.19), (3.20) using the transfer-matrix method [19. We first consider the $r \gg l$ case. We will assume that the transverse size of the wire is much less than the effective localization length of the ALS, which we will find to be $\xi_{\text {ef }} \sim \sqrt{\xi / u S}$. This means that we are indeed in the quasi-1D regime, when considering the ALS structure. We assume for definitness that the point $\mathrm{r}$ is located to the positive direction from the coordinate origin: $-L_{-}<0<r<L_{+}$. We get then:

$$
\left\langle|\psi(\boldsymbol{r})|^{2}|\psi(0)|^{2 q}\right\rangle=\frac{1}{V(S \xi)^{q}} q \int_{0}^{\infty} d z z^{q-2} W^{(1)}\left(z, \tau_{-}\right) W^{(2)}\left(z, \tau_{1}, \tau_{2}\right)
$$

where $\tau_{-}=L_{-} / \xi, \tau_{1}=r / \xi, \tau_{2}=\left(L_{+}-r\right) / \xi$; the function $W^{(1)}(z, \tau)$ is defined by eqs.(3.5)(3.7), and the function $W^{(2)}\left(z, \tau_{1}, \tau_{2}\right)$ satisfies the same equation

$$
\frac{\partial W^{(2)}\left(z, \tau_{1}, \tau_{2}\right)}{\partial \tau_{1}}=\left(z^{2} \frac{\partial^{2}}{\partial z^{2}}-z\right) W^{(2)}\left(z, \tau_{1}, \tau_{2}\right)
$$

and the boundary condition

$$
W^{(2)}\left(z, 0, \tau_{2}\right)=z W^{(1)}\left(z, \tau_{2}\right)
$$

The solution of eqs.(3.25), (3.26) is 19

$$
\begin{aligned}
& W^{(2)}\left(z, \tau_{1}, \tau_{2}\right)=2 \sqrt{z} \int_{0}^{\infty} d \nu b\left(\nu, \tau_{2}\right) K_{i \nu}(2 \sqrt{z}) e^{-\frac{1+\nu^{2}}{4} \tau_{1}} \\
& b\left(\nu, \tau_{2}\right)=\frac{\nu \sinh (\pi \nu)}{2 \pi^{2}} \int_{0}^{\infty} d t K_{i \nu}(t) W^{(1)}\left(t^{2} / 4, \tau_{2}\right)
\end{aligned}
$$

Substituting here the formula (3.7) for $W^{(1)}\left(z, \tau_{2}\right)$ and evaluating the integral over $z$, we reduce eq.(3.27) for $b\left(\nu, \tau_{2}\right)$ to the form

$$
\begin{aligned}
b\left(\nu, \tau_{2}\right) & =\frac{\nu \sinh (\pi \nu)}{16 \pi^{2}}\left|\Gamma\left(\frac{1+i \nu}{2}\right)\right|^{4}\left(1+\nu^{2}\right) \\
& +\frac{\nu \sinh (\pi \nu)}{2 \pi^{3}} \int \frac{d \nu_{1} \nu_{1}}{1+\nu_{1}^{2}} \sinh \frac{\pi \nu_{1}}{2}\left|\Gamma\left(1+i \frac{\nu+\nu_{1}}{2}\right)\right|^{2}\left|\Gamma\left(1+i \frac{\nu-\nu_{1}}{2}\right)\right|^{2} e^{-\frac{1+\nu_{1}^{2}}{4} \tau_{2}}
\end{aligned}
$$

Equations (3.24), (3.7), (3.27), (3.28) constitute the final result for the moments $\left\langle|\psi(\boldsymbol{r})|^{2}|\psi(0)|^{2 q}\right\rangle$ at $r \gg l$. Now we can restore the function $\mathcal{Q}(u, \boldsymbol{r})$ : 


$$
\begin{aligned}
\mathcal{Q}(u, \boldsymbol{r}) & \equiv\left\langle\delta\left(|\psi(0)|^{2}-u\right)|\psi(\boldsymbol{r})|^{2}\right\rangle \\
& =-\frac{1}{V \xi S} \frac{\partial}{\partial u}\left[\frac{W^{(2)}\left(u \xi S, \tau_{1}, \tau_{2}\right) W^{(1)}\left(u \xi S, \tau_{-}\right)}{u}\right], \quad r \gg l
\end{aligned}
$$

In the opposite case, $r \ll l$, we find from eq.(3.20)

$$
\mathcal{Q}(u, \boldsymbol{r})=\frac{1}{V}\left\{k_{d}(r)\left(u \frac{d^{2}}{d u^{2}}+\frac{d}{d u}\right)-\frac{d}{d u}\right\} Y_{a}(u)
$$

where the function $Y_{a}(u)$ was defined in eq.(2.3). This formula is valid for any sample, which is locally $d$-dimensional. In the case of the quasi-1D geometry we get

$$
\mathcal{Q}(u, \boldsymbol{r})=\frac{1}{V}\left\{k_{d}(r)\left(u \frac{d^{2}}{d u^{2}}+\frac{d}{d u}\right)-\frac{d}{d u}\right\}\left[W^{(1)}\left(u \xi S, \tau_{-}\right) W^{(1)}\left(u \xi S, \tau_{+}\right)\right], \quad r \ll l
$$

Substituting these results along with the formula (3.4) for $\mathcal{P}(u)$ in eq.(3.1), we can find the average density $\left\langle\left|\psi^{2}(\boldsymbol{r})\right|\right\rangle_{u}$. It is possible to check that it satisfies the normalization requirment $\int\left\langle\left|\psi^{2}(\boldsymbol{r})\right|\right\rangle_{u}=1$, as it should be. In the following two subsections, we will study the form of $\left\langle\left|\psi^{2}(\boldsymbol{r})\right|\right\rangle_{u}$ for an ALS in the insulating $(L \gg \xi)$ and the metallic $(L \ll \xi)$ limits.

\section{Insulating sample $(L \gg \xi)$.}

In this subsection, we analyze the above general results for $\left\langle\left|\psi^{2}(\boldsymbol{r})\right|\right\rangle_{u}$ in the limit of an infinite sample length. More precisely, we will assume that the distances $L_{-}, L_{+}$from the point $\boldsymbol{r}=0$ to the both edges of the sample are much larger than $\xi$, so that we can consider the limit $\tau_{-}, \tau_{+} \rightarrow \infty$. We have then

$$
W^{(1)}(z, \infty)=2 \sqrt{z} K_{1}(2 \sqrt{z})
$$

and the distribution function $\mathcal{P}(u)$ takes the form (2.14). Furthermore, eqs.(3.27), (3.28) reduce to

$$
W^{(2)}\left(z, \tau_{1}, \infty\right)=2 \sqrt{z} \int_{0}^{\infty} d \nu \frac{\nu\left(1+\nu^{2}\right)}{8} \tanh \frac{\pi \nu}{2} K_{i \nu}(2 \sqrt{z}) e^{-\frac{1+\nu^{2}}{4} \tau_{1}}
$$

Typical values for the intensity $u$ of a localized state are $u \lesssim 1 / S \xi$. We are interested however in anomalously high $u \gg 1 / S \xi$. It is seen from eqs.(3.4), (3.7), (3.29), (3.33), that this corresponds to large values $t=2 \sqrt{u S \xi} \gg 1$ of the argument of the modified Bessel functions $K_{1}(t)$ and $K_{i \nu}(t)$. The corresponding asymptotic formulae read [29]:

$$
\begin{aligned}
& K_{1}(t) \simeq \sqrt{\frac{\pi}{2 t}} e^{-t}, \quad t \gg 1 \\
& K_{i \nu}(t) \simeq \sqrt{\frac{\pi}{2 t}} \exp \left\{-t-t f\left(\frac{\nu}{t}\right)\right\}, \quad 1 \ll t, \quad \nu<t \\
& f(w)=\sqrt{1-w^{2}}+w \arcsin w-1=\frac{w^{2}}{2}+O\left(w^{4}\right)
\end{aligned}
$$

The asymptotics of the distribution function $\mathcal{P}(u)$ has the form 


$$
V \mathcal{P}(u) \simeq 4 \pi S^{3 / 2} \xi^{3 / 2} u^{-1 / 2} e^{-4 \sqrt{u S \xi}}
$$

As to the function $\mathcal{Q}(u, r)$, we consider separately three regions of the distance $r$ :

a) $r \gg \xi$, i.e. $\tau_{1}=r / \xi \gg 1$.

The integral in $(3.33)$ is then determined by the region $\nu \sim \tau_{1}^{-1 / 2}$, yielding

$$
W^{(2)}\left(z=t^{2} / 4, \tau_{1}, \infty\right) \simeq \frac{\pi^{2}}{8 \sqrt{2}} t^{1 / 2} \tau_{1}^{-3 / 2} e^{-t-\tau_{1} / 4}
$$

where we have denoted $t=2 \sqrt{z}$. Consequently,

$$
V \mathcal{Q}(u, r)=\frac{\pi^{5 / 2}}{4 u} \tau_{1}^{-3 / 2} e^{-4 \sqrt{u S \xi}-\tau_{1} / 4}
$$

b) $l \ll r \ll \xi$.

We have

$$
\begin{aligned}
W^{(2)}\left(z=t^{2} / 4, \tau_{1}, \infty\right) & \simeq \frac{1}{8} \sqrt{\frac{\pi t}{2}} e^{-t-\tau_{1} / 4} \int_{0}^{\infty} d \nu \nu\left(1+\nu^{2}\right) \tanh \frac{\pi \nu}{2} \exp \left\{-\nu^{2}\left(\frac{1}{2 t}+\frac{\tau_{1}}{4}\right)\right\} \\
& =\sqrt{\frac{\pi}{2}} t^{5 / 2} \frac{1}{\left(\tau_{1} t+2\right)^{2}} e^{-t}
\end{aligned}
$$

so that

$$
V \mathcal{Q}(u, r)=2 \pi \xi S e^{-4 \sqrt{u S \xi}} \frac{1}{\left(1+\tau_{1} \sqrt{u S \xi}\right)^{2}}
$$

c) $r \ll l$.

In this region an additional term proportional to the Friedel function $k_{d}(r)$ appears in the expression for $\mathcal{Q}(u, r)$, see eq.(3.31). We get

$$
V \mathcal{Q}(u, r)=2 \pi \xi S e^{-4 \sqrt{u S \xi}}\left[1+2 \sqrt{u S \xi} k_{d}(r)\right]
$$

Since $k_{d}(r) \sim 1 /\left(k_{F} r\right)^{d-1}$ for $r \gg k_{F}^{-1}$, the second term in square brackets is much larger than the first one at $r \ll r_{0} \sim k_{F}^{-1}(u S \xi)^{1 /[2(d-1)]}$ and is negligible at $r \gg r_{0}$.

Substituting now eqs.(3.36), (3.38), (3.40), and (3.41) in eq. (3.1), we find the following spatial structure of the ALS with $\left|\psi^{2}(0)\right|=u$ :

$$
\begin{array}{rlrl}
\left\langle\left|\psi^{2}(r)\right|\right\rangle_{u} & =\frac{\pi^{3 / 2}}{16} u^{-1 / 2} S^{-3 / 2} r^{-3 / 2} e^{-r / 4 \xi}, & & r \gg \xi \\
\left\langle\left|\psi^{2}(r)\right|\right\rangle_{u}=\frac{1}{2}\left(\frac{u}{\xi S}\right)^{1 / 2} \frac{1}{\left(1+r \sqrt{\frac{u S}{\xi}}\right)^{2}}, & & l<r \ll \xi \\
\left\langle\left|\psi^{2}(r)\right|\right\rangle_{u}=\frac{1}{2}\left(\frac{u}{\xi S}\right)^{1 / 2}\left[1+2 \sqrt{u S \xi} k_{d}(r)\right], & & r<l
\end{array}
$$

We see from eqs.(3.42), (3.43), (3.44) that the eigenfunction normalization is dominated by the region $r \sim \xi_{\text {ef }}$, where $\xi_{\text {ef }} \sim \sqrt{\xi / u S} \ll \xi$ plays the role of an effective localization length. In the region $\xi_{\text {ef }} \ll r \ll \xi$ the wave intensity falls down as $1 / r^{2}$, and transits to the 
conventional localization behavior at $r \gg \xi$. Therefore, the appearance of an anomalously high amplitude $\left|\psi^{2}(0)\right|=u \gg 1 / S \xi$ is not just a local fluctuation, but rather a kind of a cooperative phenomenon corresponding to existence of a whole region $r \lesssim \xi_{\text {ef }}$ with an unusually large amplitude $\left|\psi^{2}(r)\right|=\frac{1}{2} \sqrt{u / \xi S} \sim 1 / S \xi_{\text {ef }}$.

Let us emphasize once more that what we have calculated is the average value $\left\langle\left|\psi^{2}(r)\right|\right\rangle$ of the eigenfunction intensity with the condition $\left|\psi^{2}(0)\right|=u$. It is natural to ask now what are its fluctuations. This question is addressed in section IIIB. We will also explain there what is the reason for the "quasi-jump" of $\left\langle\left|\psi^{2}\right|\right\rangle$ from $\left|\psi^{2}(0)\right|=u$ to $\left\langle\left|\psi^{2}(r)\right|\right\rangle=\frac{1}{2} \sqrt{u / \xi S}$ at $r \sim l$.

\section{Metallic state $(L \ll \xi)$.}

We will assume that the observation point $r=0$ is located somewhere in the bulk of the sample, so that both $\tau_{-}=L_{-} / \xi$ and $\tau_{+}=L_{+} / \xi$ are of the same order of magnitude as $L / \xi=\tau_{-}+\tau_{+}$:

$$
\tau_{-}, \tau_{+} \sim L / \xi=1 / g \ll 1
$$

The distribution of eigenfunction intensities is given by eqs.(3.4), (3.7), and its behavior in various ranges of the variable $y=u V$ is indicated in eqs.(2.10)-(2.13). We will study the structure of the ALS responsible for the far asymptotics 2.13) in the region $u V \gg g$. As is seen from eqs.(3.4), (3.7), this corresponds to a large value of the argument $t=2 z^{1 / 2}=$ $2(u S \xi)^{1 / 2} \gg g$ of the modified Bessel function in eq.(3.7). Under this condition, the integral in eq.(3.7) can be evaluated via the stationary point method with use of the asymptotic expression(3.35) for the modified Bessel function:

$$
\begin{aligned}
W^{(1)}\left(z=t^{2} / 4, \tau\right)= & \frac{\tau}{\pi} \sqrt{\frac{t}{2 \pi}} e^{-t} \int_{0}^{\infty} d \nu \exp \left\{\frac{\pi \nu}{2}-\frac{\nu^{2}}{4} \tau-t f\left(\frac{\nu}{t}\right)\right\} \\
= & \frac{1}{\pi} \sqrt{2 t \tau} \exp \left\{-t+\frac{\pi^{2}}{4 \tau}-t \tilde{f}\left(\frac{\pi}{t \tau}\right)\right\}, \quad t \gg 1 / \tau \gg 1 ; \\
& \tilde{f}(w)=\frac{w^{2}}{2}+\ldots, \quad w \ll 1
\end{aligned}
$$

The distribution function $\mathcal{P}(u)$ has therefore the form

$$
\begin{aligned}
& \mathcal{P}(u)=\frac{16}{\pi^{2}} \sqrt{\frac{S \xi}{u}} \frac{\sqrt{L_{+} L_{-}}}{L} \exp \{-4 \sqrt{u \xi S}+\frac{\pi^{2} \xi}{4 L_{+}}+\frac{\pi^{2} \xi}{4 L_{-}} \\
&\left.-2 \sqrt{u \xi S}\left[\tilde{f}\left(\frac{\pi}{2 L_{+}} \sqrt{\frac{\xi}{u S}}\right)+\tilde{f}\left(\frac{\pi}{2 L_{-}} \sqrt{\frac{\xi}{u S}}\right)\right]\right\} \\
&=\frac{16}{\pi^{2}} \sqrt{\frac{S \xi}{u}} \frac{\sqrt{L_{+} L_{-}}}{L} \exp \left\{-4 \sqrt{u \xi S}+\frac{\pi^{2} \xi}{4 L_{+}}\left(1-\frac{\sqrt{\xi / u S}}{L_{+}}+\ldots\right)\right. \\
&+\left.\frac{\pi^{2} \xi}{4 L_{-}}\left(1-\frac{\sqrt{\xi / u S}}{L_{-}}+\ldots\right)\right\}
\end{aligned}
$$


This is just the formula (2.13), but written now with full accuracy with respect to the subleading factors.

To calculate $\mathcal{Q}(u, r)$, eq.(3.29), we have first to evaluate the function $W^{(2)}\left(z, \tau_{1}, \tau_{2}\right)$, eq.(3.27). The contribution to it from the first term in eq.(3.28) was calculated in the preceding subsection, where the insulating regime was considered. We will find that in the metallic regime the second term in eq.(3.28) gives a much larger contribution, so that the first one can be neglected. Substituting the second term of eq.(3.27) into (3.28), we find after simple algebraic transformations:

$$
\begin{aligned}
W^{(2)}\left(z=t^{2} / 4, \tau_{1}, \tau_{2}\right) & =\frac{t}{4 \pi} \int d \nu K_{i \nu}(t) e^{-\frac{1+\nu^{2}}{4} \tau_{1}} \nu \sinh (\pi \nu) \\
& \times \int \frac{d \nu_{1} \nu_{1}}{1+\nu_{1}^{2}} \sinh \frac{\pi \nu_{1}}{2} \frac{\nu_{1}^{2}-\nu^{2}}{\cosh \left(\pi \nu_{1}\right)-\cosh (\pi \nu)} e^{-\frac{1+\nu_{1}^{2}}{4} \tau_{2}}
\end{aligned}
$$

Analysis of the double integral in eq.(3.47) shows that one should distinguish between the two possible siuations:

$$
\text { i) } \tau_{1}+\frac{2}{t} \ll \tau_{2}^{2} \sim 1 / g^{2}
$$

This corresponds to a very large amplitude $u \sim \frac{t^{2}}{\xi S} \gg V^{-1} g^{3}$. The leading contribution to the integral in (3.47) comes from the region $\nu \gg \nu_{1}$. To check this, let us assume that $\nu \gg \nu_{1}$ and collect the exponential factors in eq.(3.47):

$$
W^{(2)}\left(z=t^{2} / 4, \tau_{1}, \tau_{2}\right) \sim \int d \nu \exp \left\{-\nu^{2} \frac{\tau_{1}}{4}-\frac{\nu^{2}}{2 t}\right\} \int d \nu_{1} \exp \left\{\frac{\pi \nu_{1}}{2}-\frac{\nu_{1}^{2} \tau_{2}}{4}\right\},
$$

so that the characteristic values of the variables are $\nu \sim\left(\tau_{1}+2 / t\right)^{-1 / 2}$ and $\nu_{1} \sim 1 / \tau_{2}$, confirming the consistency of our assumption in the considered range of parameters. Collecting all the prefactors, we get

$$
W^{(2)}\left(z=t^{2} / 4, \tau_{1}, \tau_{2}\right)=\frac{1}{2 \pi} \sqrt{\frac{t \tau_{+}}{2}} \frac{1}{\left(1 / t+\tau_{1} / 2\right)^{2}} e^{\pi^{2} / 4 \tau_{+}-t} ; \quad \tau_{+}=\tau_{1}+\tau_{2}
$$

Note that we have omitted the corrections of the type $t \tilde{f}\left(\pi / t \tau_{+}\right)$in the exponent, since they are small in the considered case $t \gg g^{2}$. Substituting eq.(3.48) in eq.(3.29), we find

$$
\mathcal{Q}(u, r)=\frac{8}{\pi^{2} t^{2}} \frac{\sqrt{L_{+} L_{-}}}{L} \frac{1}{(1 / t+r / 2 \xi)^{2}} \exp \left\{\frac{\pi^{2}}{4} \frac{\xi}{L_{+}}+\frac{\pi^{2}}{4} \frac{\xi}{L_{-}}-2 t\right\} ; \quad t=2 \sqrt{u S \xi}
$$

and finally

$$
\left\langle\left|\psi^{2}(r)\right|\right\rangle_{u}=\frac{1}{2}\left(\frac{u}{\xi S}\right)^{1 / 2} \frac{1}{\left(1+r \sqrt{\frac{u S}{\xi}}\right)^{2}}
$$

ii) $\tau_{2}^{2} \ll \tau_{1}+\frac{2}{t}$.

In this case the integrals in (3.47) are dominated by the domain $\nu_{1} \sim \nu \gg 1$. Introducing the new variable $\nu_{-}=\nu-\nu_{1}$, we reduce eq.(3.47) to the form 


$$
\begin{aligned}
W^{(2)}\left(z=t^{2} / 4, \tau_{1}, \tau_{2}\right) & =\frac{1}{8} \sqrt{\frac{t}{2 \pi}} \int d \nu d \nu_{-} \frac{\nu \nu_{-}}{\sinh \left(\pi \nu_{-} / 2\right)} \\
& \times \exp \left\{-t-t f\left(\frac{\nu}{t}\right)+\frac{\pi \nu}{2}-\frac{\nu^{2}}{4} \tau_{1}-\frac{\nu_{-}^{2}}{4} \tau_{2}+\frac{\nu \nu_{-}}{2} \tau_{2}\right\}
\end{aligned}
$$

Evaluating the integrals, we find

$$
\begin{array}{r}
W^{(2)}\left(z=t^{2} / 4, \tau_{1}, \tau_{2}\right)=\frac{1}{4 \pi} \sqrt{\frac{2 t}{\tau_{+}^{3}}}\left[\zeta\left(2,1-\frac{\tau_{1}+2 / t}{2 \tau_{+}}\right)+\zeta\left(2, \frac{\tau_{1}+2 / t}{2 \tau_{+}}\right)\right] \\
\times \exp \left\{\frac{\pi^{2}}{4 \tau_{+}}-t \tilde{f}\left(\frac{\pi}{t \tau_{+}}\right)\right\}
\end{array}
$$

where $\zeta(p, z)$ is the generalized Riemann's zeta-function:

$$
\zeta(p, z)=\sum_{k=0}^{\infty}(z+k)^{-p}
$$

Substituting this in eq.(3.29), we get

$$
\begin{aligned}
& \mathcal{Q}(u)=\frac{8 \xi}{\pi^{2} t^{2} L} \sqrt{\frac{\tau_{1}}{\tau_{+}^{3}}} {\left[\zeta\left(2,1-\frac{\tau_{1}+2 / t}{2 \tau_{+}}\right)+\zeta\left(2, \frac{\tau_{1}+2 / t}{2 \tau_{+}}\right)\right] } \\
& \times \exp \left\{\frac{\pi^{2}}{4 \tau_{+}}+\frac{\pi^{2}}{4 \tau_{-}}-2 t-t \tilde{f}\left(\frac{\pi}{t \tau_{+}}\right)-t \tilde{f}\left(\frac{\pi}{t \tau_{-}}\right)\right\}, \quad t=2 \sqrt{u S \xi}
\end{aligned}
$$

Therefore, the average local intensity of the ALS is given by

$$
\left\langle\left|\psi^{2}(r)\right|\right\rangle_{u}=\frac{1}{8 S L_{+}^{2}} \sqrt{\frac{\xi}{u S}}\left[\zeta\left(2, \frac{r+\sqrt{\xi / S u}}{2 L_{+}}\right)+\zeta\left(2,1-\frac{r+\sqrt{\xi / S u}}{2 L_{+}}\right)\right]
$$

In particular, in its "core", $r \ll L_{+}$, the ALS intensity has again the form (3.50).

Comparing eqs.(3.50), (3.54) with the result(3.43), we conclude that in its central "bump" the ALS in the metallic sample has exactly the same spatial structure as in a long (insulating) one. The condition $V u \gg g$, under which the asymptotical behavior (2.13), (3.46) is valid, acquires now a very transparent meaning. This is just the condition that the effective localization length of an ALS, $\xi_{e f}=\sqrt{\xi / u S}$ is much less than the sample size L. Indeed, $\xi_{\text {ef }} / L=\sqrt{\xi / u S L^{2}}=\sqrt{g / u V}$. In the "tail", $r \sim L \gg \xi_{e f}$, the form of the ALS intensity is slightly modified by the boundary of the sample, see eq.(3.54).

All the above calculations in this subsection were valid for the domain $r>l$. As to the region $r<l$, we can easily satisfy ourselves using eqs.(3.31) and (3.45) that the result (3.44) holds. Therefore, the "quasi-jump" of $\left\langle\left|\psi^{2}(r)\right|\right\rangle_{u}$ at $r \ll l$ has the same form, as in the insulating regime.

\section{B. Fluctuations of the eigenfunction of an anomalously localized state.}

In the section $\llbracket I I A$ we have calculated the average intensity $\left\langle\left|\psi^{2}(r)\right|\right\rangle_{u}$ of a quantum state which has an anomalously high amplitude $\left|\psi^{2}(0)\right|=u$ in a certain point $\boldsymbol{r}=0$. However, this 
average value does not give full information about the ALS. In the present subsection, we will study the fluctuations of the intensity $\left|\psi^{2}(r)\right|$ of an ALS fixed by the condition $\left|\psi^{2}(0)\right|=u$. Similarly to the subsection ПIIA (see eq.(3.47)), we express the moments $\left\langle|\psi(\boldsymbol{r})|^{2 p}|\psi(0)|^{2 q}\right\rangle$ in terms of the Green's functions:

$$
\begin{aligned}
& \left\langle|\psi(\boldsymbol{r})|^{2 p}|\psi(0)|^{2 q}\right\rangle \\
& \equiv \frac{1}{\nu_{0} V}\left\langle\sum_{\alpha}\left|\psi_{\alpha}(\boldsymbol{r})\right|^{2 p}\left|\psi_{\alpha}(0)\right|^{2 q} \delta\left(E-E_{\alpha}\right)\right\rangle \\
& =\lim _{\eta \rightarrow 0} \frac{i^{q-p}}{2 \pi \nu_{0} V} \frac{(q-1) !(p-1) !}{(q+p-2) !}(2 \eta)^{q+p-1}\left\langle G_{R}^{q}(0,0) G_{A}^{p}(\boldsymbol{r}, \boldsymbol{r})\right\rangle,
\end{aligned}
$$

The analogue of eq.(3.12) reads

$$
\begin{aligned}
G_{R}^{q}(0,0) G_{A}^{p}(\boldsymbol{r}, \boldsymbol{r}) & =\frac{i^{p-q}}{p ! q !} \int D \Phi D \Phi^{\dagger}\left(S_{1}(0) S_{1}^{*}(0)\right)^{q}\left(S_{2}(\boldsymbol{r}) S_{2}^{*}(\boldsymbol{r})\right)^{p} \\
& \times \exp \left\{i \int d \boldsymbol{r}^{\prime} \Phi^{\dagger}\left(\boldsymbol{r}^{\prime}\right) \Lambda^{1 / 2}(E+i \eta \Lambda-\hat{H}) \Lambda^{1 / 2} \Phi\left(\boldsymbol{r}^{\prime}\right)\right\}
\end{aligned}
$$

Proceeding further as in the section IIIA, we get for $r \gg l$

$$
\left\langle|\psi(\boldsymbol{r})|^{2 p}|\psi(0)|^{2 q}\right\rangle=\frac{(-1)^{p}}{2 V} \lim _{\eta \rightarrow 0}\left(2 \pi \nu_{0} \eta\right)^{q+p-1} \frac{(q-1) !(p-1) !}{(q+p-2) !} \int D Q Q_{11, b b}^{q}(0) Q_{22, b b}^{p}(\boldsymbol{r}) e^{-S\{Q\}}
$$

For the quasi-1D geometry the integral can be again evaluated via the method of [19], yielding

$$
\left\langle|\psi(r)|^{2 p}|\psi(0)|^{2 q}\right\rangle=\frac{1}{V(\xi S)^{q+p-1}} \frac{q ! p !}{(q+p-2) !} \int_{0}^{\infty} d z z^{q-2} W^{(1)}\left(z, \tau_{-}\right) W_{p}^{(2)}\left(z, \tau_{1}, \tau_{2}\right),
$$

where $W_{p}^{(2)}$ satisfies the same equation (3.25) as the function $W^{(2)} \equiv W_{1}^{(2)}$, and the boundary condition generalizing eq. (3.26):

$$
W_{p}^{(2)}\left(z, 0, \tau_{2}\right)=z^{p} W^{(1)}\left(z, \tau_{2}\right)
$$

Defining similarly to eq.(3.3),

$$
\mathcal{Q}_{p}(u, \boldsymbol{r})=\frac{1}{\nu_{0} V}\left\langle\sum_{\alpha}\left|\psi_{\alpha}(\boldsymbol{r})\right|^{2 p} \delta\left(\left|\psi_{\alpha}(0)\right|^{2}-u\right) \delta\left(E-E_{\alpha}\right)\right\rangle
$$

we get

$$
\mathcal{Q}_{p}(u, r)=\frac{p}{V(S \xi)^{p}} \int_{1}^{\infty} \frac{d v}{v}\left(1-\frac{1}{v}\right)^{p-1} \frac{\partial^{2}}{\partial u^{2}}\left[W_{p}^{(2)}\left(v u \xi S, \tau_{1}, \tau_{2}\right) W^{(1)}\left(v u \xi S, \tau_{-}\right)\right], \quad p>1
$$

For the second moment, $p=2$, this formula can be simplified 


$$
\mathcal{Q}_{2}(u, r)=2 \frac{W_{2}^{(2)}\left(u \xi S, \tau_{1}, \tau_{2}\right) W^{(1)}\left(u \xi S, \tau_{-}\right)}{V(u S \xi)^{2}}
$$

The solution to eqs.(3.25), (3.59) has the form

$$
\begin{aligned}
& W_{p}^{(2)}\left(z, \tau_{1}, \tau_{2}\right)=2 \sqrt{z} \int_{0}^{\infty} d \nu b_{p}\left(\nu, \tau_{2}\right) K_{i \nu}(2 \sqrt{z}) e^{-\frac{1+\nu^{2}}{4} \tau_{1}} \\
& b_{p}\left(\nu, \tau_{2}\right)=\frac{2 \nu \sinh (\pi \nu)}{\pi^{2}} \int_{0}^{\infty} \frac{d t}{t^{2}}\left(\frac{t^{2}}{4}\right)^{p} K_{i \nu}(t) W^{(1)}\left(t^{2} / 4, \tau_{2}\right)
\end{aligned}
$$

As we have seen above, an ALS in a metallic sample has essentially the same spatial structure as in an insulating one. Physical reason for this is very simple: an ALS with the effective localization length $\xi_{\text {ef }}$ may exist in a sample with the length $L \gg \xi_{e f}$ without essential modifications. This reason is equally valid for the fluctuations. Therefore, we can limit ourselves by studying the fluctuations in the technically simpler case of an infinitely long sample. In this case, the function $W^{(1)}$ is given by eq.(3.32), and eq.(3.64) reduces to

$$
\begin{aligned}
b_{p}\left(\nu, \tau_{2}=\infty\right) & =\frac{\nu \sinh (\pi \nu)}{4 \pi^{2}(2 p-1) !}\left|\Gamma\left(p+\frac{1+i \nu}{2}\right)\right|^{2}\left|\Gamma\left(p-\frac{1+i \nu}{2}\right)\right|^{2} \\
& =\frac{\nu \tanh (\pi \nu / 2)}{2^{4 p-1}(2 p-1) !}\left[\nu^{2}+(2 p-1)^{2}\right]\left[\nu^{2}+(2 p-3)^{2}\right]^{2} \ldots\left[\nu^{2}+1\right]^{2}
\end{aligned}
$$

The integral in eq.(3.63) is determined by $\nu \gg 1$. Therefore, for not too high moments $p$ the inequality $p \ll \nu$ is satisfied; the corresponding restriction on $p$ will be found explicitly below. We have then

$$
b_{p}(\nu, \infty) \simeq \frac{1}{(2 p-1) !}\left(\frac{\nu}{2}\right)^{4 p-1}
$$

and consequently from eq.(3.63),

$$
W_{p}^{(2)}\left(z=t^{2} / 4, \tau_{1}, \infty\right) \simeq e^{-t} \sqrt{\frac{\pi t}{2}} \frac{1}{\left(\tau_{1}+2 / t\right)^{2 p}}
$$

Substituting this in eq.(3.61), we get

$$
\mathcal{Q}_{p}(u, r) \simeq \frac{4 \pi p}{V}(S \xi)^{3 / 2-p} u^{-1 / 2} \int_{1}^{\infty} d v v^{1 / 2}\left(1-\frac{1}{v}\right)^{p-1} e^{-4 \sqrt{v u \xi S}} \frac{1}{\left(\tau_{1}+\sqrt{\frac{1}{v u \xi S}}\right)^{2 p}}
$$

For not too large $p \ll \sqrt{u \xi S}$ the integral is determined by the region $v-1 \ll 1$, because of the factor $\exp \{-4 \sqrt{v u \xi S}\}$. It can be then estimated as

$$
\begin{aligned}
\mathcal{Q}_{p}(u, r) & \simeq \frac{4 \pi p}{V}(S \xi)^{3 / 2-p} u^{-1 / 2} \frac{1}{\left(\tau_{1}+\sqrt{\frac{1}{u \xi S}}\right)^{2 p}} \int_{1}^{\infty} d v(v-1)^{p-1} e^{-4 \sqrt{v u \xi S}} \\
& =\frac{4 \pi}{V} p !(S \xi)^{3 / 2} u^{-1 / 2} e^{-4 \sqrt{u \xi S}}\left[\frac{\sqrt{u / \xi S}}{2\left(1+\tau_{1} \sqrt{u S \xi}\right)^{2}}\right]^{p}
\end{aligned}
$$


Finally, the $p$-th moment of the ALS intensity is given by

$$
\left\langle\left|\psi^{2 p}(r)\right|\right\rangle_{u}=\frac{\mathcal{Q}_{p}(u, r)}{\mathcal{P}(u)}=p !\left[\frac{\sqrt{u / \xi S}}{2(1+r \sqrt{u S / \xi})^{2}}\right]^{p}
$$

In course of the derivation we assumed that $p \ll \nu$ for typical values of $\nu$ in the integral (3.63). These values are

$$
\nu^{2} \sim \frac{p}{\tau_{1}+\frac{1}{\sqrt{u S \xi}}}
$$

so that the condition reads

$$
p \ll \frac{1}{\frac{r}{\xi}+\frac{1}{\sqrt{u S \xi}}} \equiv p_{\max }
$$

An ALS is defined by the condition $u S \xi \gg 1$, so that for $r \ll \xi$ we have $p_{\max } \gg 1$.

Comparing eq.(3.70) with eq.(3.43), we find that in this region

$$
\left\langle\left|\psi^{2 p}(r)\right|\right\rangle_{u} \simeq p !\left[\left\langle\left|\psi^{2}(r)\right|\right\rangle_{u}\right]^{p}, \quad l<r \ll \xi, \quad p \ll p_{\max }
$$

This means that the fluctuations of the eigenfunction intensity with respect to its average value are of the type

$$
\left|\psi^{2}(r)\right|=\frac{\sqrt{u / \xi S}}{2(1+r \sqrt{u S / \xi})^{2}}\left|\Phi^{2}(r)\right|, \quad l<r \ll \xi
$$

where $\left|\Phi^{2}(r)\right|$ is distributed according to the GUE law

$$
\mathcal{P}\left(\left|\Phi^{2}\right|\right) \simeq e^{-\left|\Phi^{2}\right|}
$$

The approximate result (3.74) holds for not too large $\left|\Phi^{2}\right| \ll p_{\text {max }}$.

Now we consider the fluctuations in the vicinity of the observation point, $r \ll l$. Proceeding as in the section [IIA, we find from eqs.(3.55), (3.56)

$$
\begin{aligned}
\left\langle|\psi(r)|^{2 p}|\psi(0)|^{2 q}\right\rangle=\frac{1}{V(S \xi)^{p+q-1}} & \sum_{j} \frac{p !}{j !(p-j) !} \frac{q !}{j !(q-j) !} k_{d}^{j}(r) \frac{q ! p !}{(q+p-2) !} \\
& \times \int d z z^{q+p-2} W^{(1)}\left(z, \tau_{+}\right) W^{(1)}\left(z, \tau_{-}\right)
\end{aligned}
$$

This allows one to restore the joint distribution function of $|\psi(0)|^{2}$ and $|\psi(r)|^{2}$ (see Appendix), which looks however rather cumbersome. For this reason, let us return to the expression for the moments and consider $p=2$. We find then

$$
\left\langle|\psi(r)|^{4}|\psi(0)|^{2 q}\right\rangle=\frac{2}{V(S \xi)^{q+1}}\left[1+2 q k_{d}(r)+\frac{q(q-1)}{2} k_{d}^{2}(r)\right] \int d z z^{q} W^{(1)}\left(z, \tau_{+}\right) W^{(1)}\left(z, \tau_{-}\right)
$$


Restoring the function $\mathcal{Q}_{2}(u, r)$ defined in eq.(3.60), we get

$$
\mathcal{Q}_{2}(u, r)=\frac{2}{V}\left[1-2 k_{d}(r) \frac{d}{d u} u+\frac{k_{d}^{2}(r)}{2} \frac{d^{2}}{d u^{2}} u^{2}\right]\left\{W^{(1)}\left(u S \xi, \tau_{+}\right) W^{(1)}\left(u S \xi, \tau_{-}\right)\right\}
$$

Substituting here (3.32), we find

$$
\left\langle\left|\psi^{4}(r)\right|\right\rangle_{u} \simeq k_{d}^{2}(r) u^{2}+2 u \sqrt{\frac{u}{S \xi}} k_{d}(r)\left(1-k_{d}(r)\right)+\frac{u}{2 S \xi}\left(1-k_{d}(r)\right)^{2}
$$

Therefore, the variance of $\left|\psi^{2}\right|$ is equal to

$$
\begin{aligned}
\operatorname{var}_{u}\left(\left|\psi^{2}\right|\right) & \equiv\left\langle\left|\psi^{4}(r)\right|\right\rangle_{u}-\left\langle\left|\psi^{2}(r)\right|\right\rangle_{u}^{2} \\
& \simeq \begin{cases}k_{d}(r)\left(1-k_{d}(r)\right) u \sqrt{\frac{u}{S \xi}}, & r \ll r_{0} \\
\frac{u}{4 S \xi}, & r \gg r_{0},\end{cases}
\end{aligned}
$$

with the scale $r_{0}$ as defined after eq.(3.41). We find that for $r \ll r_{0}$ the fluctuations are suppressed:

$$
\frac{\operatorname{var}_{u}\left(\left|\psi^{2}(r)\right|\right)}{\left\langle\left|\psi^{2}(r)\right|\right\rangle_{u}^{2}} \simeq \frac{1-k_{d}(r)}{k_{d}(r)} \frac{1}{\sqrt{u S \xi}} \ll 1
$$

On the other hand, for $r \gg r_{0}$ we have the GUE result

$$
\operatorname{var}_{u}\left(\left|\psi^{2}(r)\right|\right) \simeq\left\langle\left|\psi^{2}(r)\right|\right\rangle_{u}^{2}
$$

More generally, it is possible to check that for $r \gg r_{0}$ the terms containing $k_{d}(r)$ become negligible in higher moments of $\left|\psi(r)^{2}\right|$ as well, and the GUE-like fluctuations (3.72)-(3.74) take place.

Let us summarize the results of this subsection. We have found that the ALS intensity $\left|\psi^{2}(r)\right|$ exhibits in the region $r_{0} \ll r \ll \xi$ the GUE type fluctuations (3.72)-(3.74) with respect to its average value $\left\langle\left|\psi^{2}(r)\right|\right\rangle_{u}$. These fluctuations are completely analogous to those for an ordinary delocalized state in a metallic sample, see eq.2.8). The difference is that in the present case the average intensity $\left\langle\left|\psi^{2}(r)\right|\right\rangle_{u}$ is not uniform in the coordinate space. In the region $r \ll r_{0}$ the fluctuations are suppressed: $\operatorname{var}_{u}\left(\left|\psi^{2}(r)\right|\right) \ll\left\langle\left|\psi^{2}(r)\right|\right\rangle_{u}^{2}$. This is similar to what has been found in [30] for the spatial structure of an "ordinary" delocalized state with a moderately large local intensity $u=\left|\psi^{2}(0)\right|$ (when the zero-dimensional formula (2.8) holds and an ALS is not formed). This means that the intensity of a typical ALS is in this region close to the average value $\left\langle\left|\psi^{2}(r)\right|\right\rangle_{u}$, which exhibits the sharp decrease from $\left|\psi^{2}(0)\right|=u$ to $\left\langle\left|\psi^{2}(r)\right|\right\rangle_{u}=\frac{1}{2} \sqrt{\frac{u}{\xi S}}$ at $r \gg r_{0}$. It is not difficult to understand that this quasijump has the same sourse as the GUE-like fluctuations at $r \gg r_{0}$. One can ask, of course, why this short-scale fluctuation happens exactly in the center of the smooth ALS "bump" with a probability close to unity. The answer is as follows. We are studying the states with an anomalously large local intensity $u$, which is an exponentially rare event. There are two sources which may favor the formation of such a high intensity: i) formation of an ALS with a spatially non-uniform smooth envelope, and ii) short-scale GUE-like fluctuations. Both these mechanisms have exponentially small probabilities to produce an enhancement of the intensity by a large factor. The found configuration of $\left\langle\left|\psi^{2}(r)\right|\right\rangle_{u}$ (short-scale quasi-jump (3.44) on top of the smooth configuration (3.43)) represents just the optimal combination of the two mechanisms. 


\section{GENERALIZATION TO HIGHER SPATIAL DIMENSIONS.}

The calculation in the preceding section relies essentially on the quasi-1D structure of the sample, so it is not applicable for a sample of higher dimensionality. In this case, the asymptotical behavior of the distribution function $\mathcal{P}(u)$ was studied by Fal'ko and Efetov [21]. These authors used eqs.(2.4), (2.6) and applied the saddle-point method suggested for the supersymmetric $\sigma$-model by Muzykantskii and Khmelnitskii [22]. The advantage of this method is that it is applicable for any spatial dimension $d$. It was conjectured in [22, 21] that the saddle point solution mimics the spatial form of the ALS. Having at our disposal the exact solution for the quasi-1D case, we can check the accuracy of the saddle-point method and of this conjecture. Comparing the form of $\mathcal{P}(u)$ found in the quasi-1D case by the saddle-point method [21] with the result of the exact solution (3.46), we find a very good

agreement between them. Furthermore, the saddle-point configuration in the quasi-1D case has the form [21,24]

$$
\frac{\lambda_{1}(r)}{\lambda_{1}(0)} \equiv e^{\theta(r)-\theta(0)}=\frac{1}{\left(1+r \sqrt{\frac{u}{2 \pi \nu_{0} D}}\right)^{2}} ; \quad 0<r \ll L_{+}
$$

Here $\lambda_{1}=e^{\theta} / 2$ is the eigenvalue of the $Q$-matrix which has been introduced after eq.(2.2). Comparing eq.(4.1) with eqs.(3.43), (3.50), we see that the saddle-point solution nicely reproduces the average intensity of the ALS, $\left\langle\left|\psi^{2}(r)\right|\right\rangle_{u}$ for $r>l$, up to an overall normalization factor. Being encouraged by this agreement, we will now use the results of the saddle-point study to describe the structure of the ALS in $d=2,3$.

\section{A. 2D geometry.}

For a 2D disk-shaped sample of a radius $L$ with the high amplitude point $\boldsymbol{r}=0$ in the center of the disk, the saddle-point solution was found to have the form [21]

$$
e^{\theta(r)-\theta(0)}=\left(\frac{r}{l_{*}}\right)^{-2 \mu}\left\{1-\frac{l_{*}^{2} u}{8(1-\mu)^{2} \pi \nu_{0} D}\left(\frac{r}{l_{*}}\right)^{2-2 \mu}\right\}^{-2} \approx\left(\frac{r}{l_{*}}\right)^{-2 \mu}, \quad r \geq l_{*}
$$

where the exponent $0<\mu<1$ depends on $u$ and satisfies the equation

$$
\left(\frac{L}{l_{*}}\right)^{2 \mu}=\frac{2-\mu}{8 \mu(1-\mu)^{2}} \frac{L^{2} u}{\pi \nu_{0} D}
$$

We are interested in the asymptotic region $u L^{2} \gg \pi \nu_{0} D \ln ^{-1}(L / l)$, where the distribution of the eigenfunction intensity is given by eq.(2.20), and an ALS is formed. Then the exponent $\mu$ can be approximated as

$$
\mu \simeq \frac{\ln \left(\frac{L^{2} u}{2 \pi \nu_{0} D} \ln \frac{L}{l_{*}}\right)}{2 \ln \left(L / l_{*}\right)}
$$


The lower cut-off scale $l_{*}$ appears in eq.(4.2) because of the restriction of the diffusion approximation on the momenta $q$ of the $\sigma$-model field: $q<l^{-1}$. Correspondingly, it is determined by the condition [22 24]

$$
\left.\frac{d}{d r} \theta(r)\right|_{r=l_{*}} \sim l^{-1},
$$

which yields $l_{*} \sim \mu l$, so that $l_{*}$ differs from $l$ by the logarithmic factors (4.4) only.

Normalizing properly the expression (4.2), we find that the average ALS density for $r>l_{*}$ is equal to

$$
\left\langle\left|\psi^{2}(r)\right|\right\rangle_{u}=\frac{u}{4 \pi^{2} \nu_{0} D \mu}\left(\frac{r}{l_{*}}\right)^{-2 \mu}\left\{1-\frac{l_{*}^{2} u}{8(1-\mu)^{2} \pi \nu_{0} D}\left(\frac{r}{l_{*}}\right)^{2-2 \mu}\right\}^{-2}, \quad r \geq l_{*}
$$

The saddle-point calculation of Ref. 21] assumes that $\theta(r)$ is constant for $r<l_{*}$, so that eq. (4.5) gives $\left\langle\left|\psi^{2}(r)\right|\right\rangle_{u} \simeq \frac{u}{4 \pi^{2} \nu_{0} D \mu}$ in this region. However, for very small $r<l$ the average intensity $\left\langle\left|\psi^{2}(r)\right|\right\rangle_{u}$ changes sharply, as we have seen in the quasi-1D case. Indeed, in this domain the function $\mathcal{Q}(u, r)$ is given by eq.(3.30), where $Y_{a}(u)$ has the following behavior 21

$$
Y_{a}(u) \sim \exp \left\{-\pi^{2} \nu_{0} D \frac{\ln ^{2}\left(\frac{V u}{2 \pi^{2} \nu_{0} D} \ln \frac{L}{l_{*}}\right)}{\ln \left(L / l_{*}\right)}\right\}
$$

Using now eq.(3.30) for $\mathcal{Q}(u, r)$ and eq.(2.4) for $\mathcal{P}(u)$, we get

$$
\left\langle\left|\psi^{2}(r)\right|\right\rangle_{u} \equiv \frac{\mathcal{Q}(u, r)}{\mathcal{P}(u)}=\left[1-k_{2}(r)+A(u) k_{2}(r)\right]\left\langle\left|\psi^{2}\left(r=l_{*}\right)\right|\right\rangle_{u}, \quad r<l_{*}
$$

Here the height of the quasi-jump is given by

$$
A(u) \simeq-u \frac{d}{d u} \ln Y_{a}(u) \simeq \frac{2 \pi^{2} \nu_{0} D}{\ln \left(L / l_{*}\right)} \ln \left(\frac{V u}{2 \pi^{2} \nu_{0} D} \ln \frac{L}{l_{*}}\right) \simeq 4 \pi^{2} \nu_{0} D \mu
$$

Combining eqs.(4.5), (4.7), and (4.8), we get

$$
\left\langle\left|\psi^{2}(r)\right|\right\rangle_{u}=\frac{u}{A(u)}\left[1-k_{2}(r)+k_{2}(r) A(u)\right], \quad r<l_{*}
$$

In particular, at $r=0$ we find $\left\langle\left|\psi^{2}(r)\right|\right\rangle_{u}=u$, that shows the perfect consistency of the whole procedure.

\section{B. 3D geometry.}

Now, we consider the 3D case. The saddle-point configuration has the form [21,24]

$$
\theta(r)-\theta(0)=C\left(\frac{l_{*}}{r}-1\right), \quad r \geq l_{*}
$$


where the coefficient $C$ is defined by the condition

$$
C e^{C} \sim \frac{u V}{\nu_{0} D l_{*}}
$$

and $l_{*}=C l$ is the cut-off length scale which has the same origin as in $2 \mathrm{D}$. We are interested in the asymptotic region $u V \gg \nu_{0} D l$, where eq.(4.11) yields

$$
\begin{aligned}
e^{C} & \sim \frac{u V}{g(l) \ln ^{2}[u V / g(l)]}, \\
l_{*} & \sim l \ln [u V / g(l)],
\end{aligned}
$$

where $g(l) \sim\left(k_{F} l\right)^{2}$ is the conductance on the scale of order of the mean free path $l$. The optimal configuration(4.10) has the form

$$
e^{\theta(r)-\theta(0)}=\left\{\frac{u V}{g(l) \ln ^{2}[u V / g(l)]}\right\}^{l_{*} / r-1}, \quad r \geq l_{*}
$$

This configuration determines the asymptotical behavior of the functions $Y_{a}(u), \mathcal{P}(\sqcap)$ at $V u \gg g(l)$ :

$$
\mathcal{P}(u) \sim Y_{a}(u) \sim \exp \left\{- \text { const } g(l) \ln ^{3} \frac{V u}{g(l)}\right\}
$$

Note that in order to fix the numerical coefficient in eq.4.15), one has to go beyond the long-wave-length $\sigma$-model approximation. Such a "ballistic" generalization of the $\sigma$-model was recently suggested in Ref. [33]. The height of the quasi-jump $A(u)$ is found from eq.(4.8) to be

$$
A(u) \sim g(l) \ln ^{2} \frac{V u}{g(l)}
$$

Thus, the density of ALS is

$$
\left\langle\left|\psi^{2}(r)\right|\right\rangle_{u}=\frac{u e^{\theta(r)-\theta(0)}}{A(u)} \sim \frac{1}{V}\left[\frac{u V}{g(l) \ln ^{2} \frac{u V}{g(l)}}\right]^{l_{*} / r}, \quad r \geq l_{*}
$$

The quasi-jump at small $r$ is given again by eq.(4.9), with $k_{2}(r)$ replaced by $k_{3}(r)$.

The value of $\left\langle\left|\psi^{2}(r)\right|\right\rangle_{u}$ given by eq. (4.17) exceeds considerably the average density of a delocalized state, $\left\langle\left|\psi^{2}\right|\right\rangle=1 / V$ at $r \lesssim l_{* *}$, where

$$
l_{* *} \sim l_{*} \ln \frac{u V}{g(l)} \sim l \ln ^{2} \frac{u V}{g(l)} .
$$

For larger distances, $r \gtrsim l_{* *}$, we have $\left\langle\left|\psi^{2}(r)\right|\right\rangle_{u} \simeq 1 / V$. Therefore, in contrast to quasi-1D and 2D systems, where an ALS formation is a redistribution of the eigenfunction intensity in the whole sample, in 3D it constitutes just a "bump" with the extent of order of $l_{* *}$, on top of the usual background density $\left\langle\left|\psi^{2}\right|\right\rangle=1 / V$. 


\section{Fluctuations.}

Let us now discuss briefly the fluctuations of the ALS intensity. At small $r \ll l$, we find in full analogy with eqs.(3.78)-(3.81):

$$
\left\langle\left|\psi^{4}(r)\right|\right\rangle_{u} \simeq u^{2}\left[k_{d}^{2}(r)+\frac{4}{A(u)}\left(1-k_{d}(r)\right) k_{d}(r)+\frac{2}{A^{2}(u)}\left(1-k_{d}(r)\right)^{2}\right],
$$

and consequently,

$$
\frac{\operatorname{var}_{u}\left(\left|\psi^{2}(r)\right|\right)}{\left\langle\left|\psi^{2}(r)\right|\right\rangle_{u}^{2}} \simeq \begin{cases}2 \frac{1-k_{d}(r)}{k_{d}(r) A(u)}, & r \ll r_{0} \\ 1, & r \gg r_{0}\end{cases}
$$

Here $r_{0}$ is the characteristic spatial scale of the quasi-jump, determined by the condition $k_{d}(r) A(u) \sim 1$. In 2D, using eqs.(3.22) and (4.8), we find

$$
r_{0} \sim l \frac{\ln \left(\frac{V u}{2 \pi^{2} \nu_{0} D} \ln (L / l)\right)}{\ln (L / l)}
$$

In 3D the analogous calculation would give $r_{0} \sim l \ln [V u / g(l)]>l$. This means that in fact $r_{0} \sim l$, because of the exponential decrease of $k_{d}(r) \sim e^{-r / l}$ at $r>l$, which was not taken into account in eqs.(3.22), (3.23). We see from eq. (4.19) that at $r \ll r_{0}$ the fluctuations are relatively weak, $\operatorname{var}_{u}\left(\left|\psi^{2}(r)\right|\right) \ll\left\langle\left|\psi^{2}(r)\right|\right\rangle_{u}^{2}$. In the opposite limit, $r \gg r_{0}$, the fluctuations are expected to have essentially the GUE statistics, similarly to what we have found for the quasi-1D sample geometry, see eqs.(3.72)-(3.74).

\section{ASYMPTOTIC BEHAVIOR OF DISTRIBUTIONS OF VARIOUS QUANTITIES, AND THEIR INTERRELATIONSHIP.}

In the preceding sections, we have considered the spatial structure of ALS responsible for the asymptotic behavior of the distribution of eigenfunction intensity, $\mathcal{P}(u)$. One can consider, however, other quantities, large values of which indicate in some sense a stronger localization of an eigenfunction, so that the asymptotic behavior of corresponding distribution function is also related to a kind of ALS. An illustrative example of such a quantity is the inverse partcipation ratio (IPR) $I_{2}=\int d^{d} r\left|\psi^{4}(\boldsymbol{r})\right|$. Let us consider the case of an infinite quasi-one-dimensional sample, for which the distribution function $\mathcal{P}\left(I_{2}\right)$ was calculated exactly in Ref. [31,19]. It has the following asymptotic behavior:

$$
\mathcal{P}\left(I_{2}\right) \sim \exp \left\{-\frac{\pi^{2}}{2} \xi S I_{2}\right\}
$$

where we omitted preexponential factors. Let us suppose that the asymptotic form of $\mathcal{P}\left(I_{2}\right)$ is determined by the same anomalously localized sates, which control the asymptotic behavior of $\mathcal{P}(\sqcap)$. The intensity of such an ALS was found in Sec.币I to be

$$
\left|\psi^{2}(\boldsymbol{r})\right|=\left|\psi^{2}(r)\right|_{\text {Smooth }}\left|\Phi^{2}(\boldsymbol{r})\right|, \quad r \ll \xi
$$


where

$$
\left|\psi^{2}(r)\right|_{\text {Smooth }}=\frac{1}{2 S} \frac{\xi_{e f}}{\left(r+\xi_{e f}\right)^{2}}, \quad \xi_{e f}=\sqrt{\xi / u S}
$$

and $\left|\Phi^{2}(\boldsymbol{r})\right|$ exhibits the GUE-like fluctuations (3.74). The distribution $\mathcal{P}(u)$ behaves asymptotically as $\mathcal{P}(u) \sim \exp \{-4 \sqrt{u S \xi}\}$; here the factor $\exp \{-2 \sqrt{u S \xi}\}$ is the GUE probability of the quasi-jump in the vicinity of $r=0$ with $\left|\Phi^{2}(0)\right|=2 \sqrt{u S \xi}$. The remaining $\exp \{-2 \sqrt{u S \xi}\}$ factor is therefore the weight of the envelope configuration $\left|\psi^{2}(r)\right|_{\text {smooth. The corresponding }}$ IPR is equal to $I_{2}=(1 / 3) \sqrt{u / S \xi}$. Thus, assuming that this ALS determines the asymptotics of the IPR distribution $\mathcal{P}\left(I_{2}\right)$, we would get

$$
\mathcal{P}\left(I_{2}\right) \sim \exp \left\{-6 \xi S I_{2}\right\}
$$

This result has the same exponential form, as the correct asymptotics (5.1), but with a larger numerical coefficient in the exponent. The explanation for this discrepancy is the following: an ALS, which is optimal for maximizing the local amplitude $u=\left|\psi^{2}(0)\right|$, does not optimize the IPR. A detailed study, which will be published elsewhere 32], shows that the ALS, which determine the asymptotics of the IPR distribution, have the following spatial shape:

$$
\left|\psi^{2}(r)\right|_{\text {smooth }}=\frac{1}{\pi S} \frac{\xi_{e f}}{r^{2}+\xi_{e f}^{2}}, \quad \xi_{e f}=\frac{1}{\pi I_{2} S},
$$

that is different from eq.(5.3). Thus, the spatial form of an ALS depends on the specific physical quantity (local amplitude, IPR, ... ), for which it represents an optimal fluctuation. In the remaining part of this section, we consider from this point of view the distributions of LDOS and of the relaxation times in open metallic samples, which have been studied via the supersymmetry approach in Refs. [22,23] and Ref. [24], respectively.

\section{A. Quasi-1D geometry.}

\section{Distribution of relaxation times.}

The long-time dispersion of the average conductance has the LN form 22]

$$
G(t) \sim \exp \left\{-g \ln ^{2} \frac{t \Delta}{\ln (t \Delta)}\right\} ; \quad t \gg \Delta^{-1},
$$

where $\Delta=1 / \nu_{0} L S$ is the mean level spacing and $g=2 \pi \nu_{0} D S / L$ is the dimensionless conductance. We represent eq. (5.6) as a superposition of the simple relaxation processes with mesoscopically distributed relaxation times [2]:

$$
G(t) \sim \int d t_{\phi} e^{-t / t_{\phi}} \mathcal{P}\left(t_{\phi}\right)
$$

The distribution function $\mathcal{P}\left(t_{\phi}\right)$ then behaves as follows:

$$
\mathcal{P}\left(t_{\phi}\right) \sim \exp \left\{-g \ln ^{2}\left(g \Delta t_{\phi}\right)\right\} ; \quad t_{\phi} \gg \frac{1}{g \Delta}
$$


This can be easily checked by substituting eq.(5.8) into eq.(5.7) and calculating the integral via the sationary point method; the stationary point equation being

$$
2 g t_{\phi} \ln \left(g \Delta t_{\phi}\right)=t
$$

Note that $t_{D}^{-1} \equiv g \Delta$ is the inverse characteristic time of diffusion through the sample (Thouless energy), or in other words, the typical width of a level of an open system. The formula (5.8) concerns therefore the states with anomalously small widths in the energy space. The corresponding saddle-point configuration is found from the requirment of providing a minimum to the action

$$
\mathcal{S}=-\frac{\pi \nu, \mathcal{D}}{\triangle} \int \Gamma^{\lceil} \nabla \operatorname{Str}(\nabla \mathcal{Q})^{\epsilon}=\frac{\pi \nu, \mathcal{D}}{\epsilon} \int \Gamma^{\lceil} \nabla(\nabla \theta)^{\epsilon}
$$

with the additional restriction

$$
\int d^{d} r(\cosh \theta-1)=\frac{t}{\pi \nu_{0}},
$$

and the boundary condition on the boundary with leads $\left.\theta\right|_{\text {leads }}=0$. For a quasi-1D sample of the length $L$, the solution can be approximated as [22]:

$$
\theta(r) \simeq \theta_{0}\left(1-\frac{2|r|}{L}\right), \quad-\frac{L}{2}<r<\frac{L}{2},
$$

where $\theta_{0}$ satisfies the equation

$$
e^{\theta_{0}}=\frac{2}{\pi} t \Delta \theta_{0}
$$

so that

$$
\theta_{0} \simeq \ln \left(\frac{2}{\pi} t \Delta \ln (t \Delta)\right) \simeq \ln \left(\frac{4}{\pi} g \Delta t_{\phi} \ln ^{2}\left(g \Delta t_{\phi}\right)\right)
$$

Relying on our previous experience, we believe that the smoothed intensity of the corresponding state is $\left|\psi^{2}(r)\right|_{\text {smooth }} \propto e^{\theta(r)}$, as was stated in Ref. 222]. Normalizing it by the condition $\int\left|\psi^{2}(\mathrm{r})\right|=1$, we get

$$
\left|\psi^{2}(r)\right|_{\text {smooth }}=\frac{\theta_{0}}{S L} e^{-2 \theta_{0}|r| / L}
$$

Thus, the ALS, which gives a minimum to the level width $t_{\phi}^{-1}$, has an exponential shape (5.15), (5.14).

\section{Distribution of local density of states.}

Now, let us consider the distribution $\mathcal{P}(\rho)$ of LDOS. Typically, in an open metallic sample the LDOS $\rho(E, \boldsymbol{r})$ is given by a superposition of $\sim 1 / t_{D} \Delta=g$ adjacent levels, since their widths are of order of $1 / t_{D}$. However, we can expect that for $\rho$ much greater than its average 
value $\nu_{0}$, the asymptotic form of $\mathcal{P}(\rho)$ is determined by a probability to have a single narrow resonance, which gives this value of LDOS $\rho(E, \boldsymbol{r})$. The most favorable situation happens when the resonance is located around the point $\boldsymbol{r}$ in the real space and around the energy $E$ in energy space. The LDOS provided by such a resonance is expected to be:

$$
\rho_{A L S}=\left|\psi^{2}(\boldsymbol{r})\right| \frac{2 t_{\phi}}{\pi},
$$

where $t_{\phi}^{-1}$ is the resonance width. Thus, the optimal fluctuation shoul provide now a maximum to the product of the local amplitude $u=\left|\psi^{2}(\boldsymbol{r})\right|$ and the inverse level width $t_{\phi}$. Since the distribution $\mathcal{P}\left(t_{\phi}\right)$, eq.(5.8), decays much slower than $\mathcal{P}(u)$, eq.(3.36), one should expect the asymptotic behavior of $\mathcal{P}(\rho)$ to be mainly determined by $\mathcal{P}\left(t_{\phi}\right)$. Indeed, it was found in Ref. 24 that $\mathcal{P}(\rho)$ has a $\mathrm{LN}$ form, similar to that of $\mathcal{P}\left(t_{\phi}\right)$ :

$$
\mathcal{P}(\rho) \sim \exp \left\{-\frac{\xi}{4}\left(\frac{1}{L_{+}}+\frac{1}{L_{-}}\right) \ln ^{2}\left(\rho / \nu_{0}\right)\right\},
$$

where, as before, $L_{+}$and $L_{-}$are the distances from the observation point $r=0$ to the sample edges. The corresponding saddle-point configuration reads:

$$
e^{\theta(r)} \simeq \begin{cases}\left(\rho / \nu_{0}\right)^{1-r / L_{+}}, & r>0 \\ \left(\rho / \nu_{0}\right)^{1-|r| / L_{-}}, & r<0\end{cases}
$$

If we put the observation point in the middle of the sample, $L_{+}=L_{-}=L / 2$, the configuration (5.18) acquires the same form as the optimal configuration (5.12) for the relaxation time $t_{\phi}$. The corresponding values of $t_{\phi}$ and $\rho$ are related as follows:

$$
\frac{4}{\pi} g \Delta t_{\phi} \ln ^{2}\left(g \Delta t_{\phi}\right)=\rho / \nu_{0}
$$

or, expressing $t_{\phi}$ through $\rho$,

$$
t_{\phi}=\frac{\pi \rho}{4 g \Delta \nu_{0} \ln ^{2}\left(\rho / \nu_{0}\right)}
$$

Now, we calculate the value of the local amplitude $\left|\psi^{2}(0)\right|$ for an ALS corresponding to the cofiguration (5.18). First, its smoothed intensity is given by

$$
\left|\psi^{2}(r)\right|_{\text {smooth }}=\mathcal{N}^{-1} e^{\theta(r)}=\frac{\ln \left(\rho / \nu_{0}\right)}{V}\left(\frac{\rho}{\nu_{0}}\right)^{-2|r| / L} .
$$

Second, the quasi-jump induced by the GUE-type fluctuations gives an additional factor, which can be found in the same way as prescribed by eq.(3.30):

$$
\left.A(\rho)=-\rho \frac{\partial}{\partial \rho} \ln \mathcal{P}(\rho)=\in\right\} \ln \left(\rho / \nu_{l}\right)
$$

Combining eqs.(5.20), (5.21), and (5.22), we can compute the LDOS (5.16) determined by this resonance state: 


$$
\rho_{A L S}(E, 0)=\left|\psi^{2}(0)\right|_{\text {Smooth }} \cdot A(\rho) \cdot \frac{2 t_{\phi}}{\pi}=\frac{\ln \left(\rho / \nu_{0}\right)}{V} \cdot 2 g \ln \left(\rho / \nu_{0}\right) \cdot \frac{\rho V}{2 g \ln ^{2}\left(\rho / \nu_{0}\right)}=\rho
$$

We have explicitly checked therefore that the LDOS $\rho$ is indeed determined by a single ALS, smoothed intensity of which is given by eq.(5.21). There are three sources of the enhancement of LDOS: i) amplitude of the smooth envelope of the wave function, ii) the short-scale GUE "bump", and iii) the inverse resonance width. They are represented by the three factors in eq.(5.23), respectively. Note that the calculated $\rho_{A L S}$ reproduces the value of $\rho$ with an amazing accuracy (including logarithmic factors and even the numerical coefficient).

\section{Distribution of global density of states.}

Finally, we discuss the contribution of ALS to the asymptotical behavior of the distribution function $\mathcal{P}(\nu)$ of the global density of states (DOS),

$$
\nu(E)=\frac{1}{V}\left\langle\sum_{\alpha} \delta\left(E-E_{\alpha}\right)\right\rangle=\frac{1}{V} \int d^{d} r \rho(E, r)
$$

A resonance state with an energy $E$ and width $t_{\phi}^{-1}$ gives a following contribution to $\nu(E)$ :

$$
\nu_{A L S}(E)=\frac{2}{\pi} \frac{t_{\phi}}{V}=\frac{2}{\pi} t_{\phi} \Delta \nu_{0}
$$

Thus, if we assume that the asymptotic behavior of $\mathcal{P}(\nu)$ is determined by isolated (in energy space) anomalously localized states, it will have the form:

$$
\mathcal{P}(\nu) \sim \mathcal{P}\left(t_{\phi}=\frac{\pi \nu}{2 \Delta \nu_{0}}\right) \sim \exp \left\{-g \ln ^{2}\left(g \nu / \nu_{0}\right)\right\}
$$

We will see below that an analogous procedure in 2D leads to a result for $\mathcal{P}(\nu)$ which is in full agreement with the renormalization group calculation of Altshuler, Kravtsov and Lerner [2].

\section{B. 2D geometry.}

\section{Distribution of relaxation times.}

Distribution of relaxation times $t_{\phi}$ has the form [23]

$$
\mathcal{P}\left(t_{\phi}\right) \sim \begin{cases}\left(t_{\phi} / t_{D}\right)^{-4 \pi g}, & t_{D} \ll t_{\phi} \ll t_{D}\left(\frac{L}{l}\right)^{2} \\ \exp \left\{-\frac{\pi g}{2} \frac{\ln ^{2}\left(t_{\phi} / \tau\right)}{\ln (L / l)}\right\}, & t_{\phi} \gg t_{D}\left(\frac{L}{l}\right)^{2}\end{cases}
$$

with $g=2 \pi \nu_{0} D$ being the dimensionless conductance of a 2D square. An ALS corresponding to the first regime, $t_{D} \ll t_{\phi} \ll t_{D}(L / l)^{2}$, has the following spatial structure: 


$$
\left|\psi^{2}(r)\right|_{\text {smooth }}=\mathcal{N}^{-1} e^{\theta(r)}=\frac{1}{16 \pi D t_{\phi}} \frac{1}{\left[(r / L)^{2}+L^{2} /\left(16 D t_{\phi}\right)\right]^{2}},
$$

so that it has an effective localization length $\xi_{e f} \sim L\left(t_{D} / t_{\phi}\right)^{1 / 2}$, with the intensity decreasing as $1 / r^{4}$ outside the region of the extent $\xi_{e f}$. As to the ultra-long-time region, $t_{\phi} \gg t_{D}(L / l)^{2}$, the saddle-point solution reads:

$$
e^{\theta(r)}=\frac{(r / L)^{\gamma_{t}-2}}{\left[(r / L)^{\gamma_{t}}+\frac{\gamma_{t}+2}{\gamma_{t}-2}\left(l_{*}^{(t)} / L\right)^{\gamma_{t}}\right]^{2}} ; \quad l_{*}^{(t)} \leq r \leq L,
$$

where

$$
l_{*}^{(t)}=\gamma_{t} l ; \quad \gamma_{t} \simeq \frac{\ln \left(t_{\phi} / t_{D}\right)}{\ln (R / l)}
$$

Now, $l_{t}$ plays a role of an effective localization length, and the intensity shows the following behavior:

$$
\left|\psi^{2}(r)\right| \sim \frac{1}{l_{*}^{(t) 2}}\left(\frac{r}{l_{*}^{(t)}}\right)^{-\gamma_{t}-2}, \quad l_{*}^{(t)} \leq r \leq L
$$

\section{Distribution of local density of states.}

Distribution of LDOS, $\mathcal{P}(\rho)$, has the following asymptotics [24]:

$$
\mathcal{P}(\rho) \sim \exp \left\{-\frac{\pi^{2} \nu_{0} D \ln ^{2} \rho}{\ln \left(L / l_{*}^{(\rho)}\right)}\right\} .
$$

The corresponding saddle-point solution reads

$$
e^{\theta(r)} \simeq \frac{\rho}{\nu_{0}}\left(\frac{l_{*}^{(\rho)}}{r}\right)^{\gamma_{\rho}}
$$

where $l_{*}^{(\rho)}=\gamma_{\rho} l$, and

$$
\gamma_{\rho}=\frac{\ln \left(\rho / \nu_{0}\right)}{\ln \left(L / l_{*}^{(\rho)}\right)} .
$$

Normalizing it, we get the following ALS intensity at $r \geq l_{*}^{(\rho)}$ :

$$
\left|\psi^{2}(r)\right|_{\text {smooth }} \simeq \begin{cases}\left(2-\gamma_{\rho}\right) \frac{1}{V} \frac{\rho}{\nu_{0}}\left(\frac{l_{*}^{(\rho)}}{r}\right)^{\gamma_{\rho}} ; & \gamma_{\rho}<2 \\ \left(\gamma_{\rho}-2\right) \frac{1}{\pi l_{*}^{(\rho) 2}}\left(\frac{l_{*}^{(\rho)}}{r}\right)^{\gamma_{\rho}} ; & \gamma_{\rho}>2\end{cases}
$$

The value of the GUE-type quasi-jump is 


$$
A(\rho)=-\rho \frac{\partial}{\partial \rho} \ln \mathcal{P}(\rho)=\in \gamma_{\rho} \pi^{\epsilon} \nu \mathcal{D}
$$

To estimate the escape time from this resonance state, we note that its power-law decay, $\left|\psi^{2}(r)\right| \propto\left(r / l_{*}^{(\rho)}\right)^{-\gamma_{\rho}}$, is similar to that of the ALS optimizing the relaxation time, $\left|\psi^{2}(r)\right| \propto$ $\left(r / l_{*}^{(t)}\right)^{-\gamma_{t}-2}$. This allows us to identify $\gamma_{\rho}=\gamma_{t}+2$, so that

$$
t_{\phi} / t_{D} \sim \begin{cases}\frac{\rho}{\nu_{0}}\left(\frac{l}{L}\right)^{2}, & \gamma_{\rho}>2 \\ 1, & \gamma_{\rho}<2\end{cases}
$$

up to logarithmic prefactors depending on $\gamma_{\rho}$ and $\gamma_{t}$. Substituting eqs.(5.34), (5.35), and (5.36) in eq.(5.16), we get $\rho_{A L S} \sim \rho$. Thus, we have checked that the ALS determined by the saddle-point solution (5.33) indeed provides the value of LDOS which is equal (within the accuracy of our consideration) to $\rho$. This confirms an assumption that an anomalously large value of $\rho$ is typically governed by a single ALS with the spatial structure described by the corresponding saddle point configuration. Like in the quasi-1D case, the enhancement of $\rho$ is determined by the product of three factors: $\left|\psi^{2}(r)\right|_{\text {smooth }}, A(\rho)$, and $t_{\phi} / t_{D}$, represented by eqs. (5.34), (5.35), and (5.36), respectively.

\section{Distribution of global density of states.}

Now, we consider the contribution of ALS to the asymptotics of the distribution of global DOS, which can be estimated according to eq.(5.26) as follows:

$$
\mathcal{P}(\nu) \sim \mathcal{P}\left(t_{\phi}=\frac{\pi \nu}{2 \Delta \nu_{0}}\right) \sim \begin{cases}\left(g \nu / \nu_{0}\right)^{-4 \pi g}, & \frac{\nu}{\nu_{0}} \ll \frac{1}{g}\left(\frac{L}{l}\right)^{2} \\ \exp \left\{-\frac{\pi g}{2} \frac{\ln ^{2}\left(\nu / \nu_{0} \Delta \tau\right)}{\ln (L / l)}\right\}, & \frac{\nu}{\nu_{0}} \gg \frac{1}{g}\left(\frac{L}{l}\right)^{2}\end{cases}
$$

The far LN asymptotic tail in eq.(5.37) is in full agreement with the RG calculation by Altshuler, Kravtsov, and Lerner [2]. We find also an intermediate power-law behavior, which could not be obtained from the study of cumulants in Ref. [2]. We note, however, that this power-law form is fully consistent with the change of the behavior of cumulants $\left\langle\left\langle\nu^{n}\right\rangle\right\rangle$ at $n \sim \pi g$ discovered in [2].

Finally, taking into account the close similarity between the cumulants of DOS and of conductance [2], it is natural to suppose that the distribution function of conductance in a 2D metallic system has essentially the same behavior (5.37), with an intermediate powerlaw regime. This hypothesis, which would be fully consistent with a power-law asymptotic behavior of conductance distribution function on the mobility edge suggested by Shapiro [34], needs however further verification.

\section{3D geometry.}

As has been already mentioned in Sec.IV, in three dimensions the ALS are in fact not localized. Their intensity just shows a relatively "narrow" bump on top of the usual average 
value $\left|\psi^{2}\right|=1 / V$. Comparing the results of Sec.IV and of Refs. [23,24], we find that the spatial shape of these "bumps" is the same for the states, which are optimal for all the distributions $\mathcal{P}(u), \mathcal{P}(\rho)$, and $G(t)$. Namely, it has the form

$$
\left|\psi^{2}(r)\right|_{\text {smooth }} \sim \frac{1}{V} \exp \left\{C_{i} \frac{l}{r} \ln ^{2} Z_{i}\right\}, \quad i=u, \rho, t
$$

where

$$
\begin{aligned}
Z_{u} & =\frac{u V}{\left(k_{F} l\right)^{2}}, \\
Z_{\rho} & =\rho / \nu_{0}, \\
Z_{t} & =\frac{t}{\tau\left(k_{F} l\right)^{2}},
\end{aligned}
$$

and $C_{i}$ are numerical constants of order of unity. The corresponding distributions have one and the same, log-cube-exponential, form:

$$
\mathcal{P}(i) \sim \exp \left\{-\operatorname{const}\left(k_{F} l\right)^{2} \ln ^{3} Z_{i}\right\}
$$

Note that formation of a large value of $\operatorname{LDOS} \rho(E, r)$ cannot be explained in 3D as a contribution of a single ALS. Indeed, in a metallic sample LDOS is typically provided by a number of levels of order of $g \sim k_{F}^{2} l L$. In order that a single level might give such (or even larger) a value of LDOS, it should has a local amplitude (or, alternatively, an inverse width in energy space) enhanced by a factor of $g$. However, in contrast to the quasi-1D and 2D situations, $g$ does not enter the asymptotics (5.39), which do not depend on the system size $L$. Therefore, a high value of LDOS is in 3D typically due to contribution of a large number $(\propto L / l)$ of adjacent levels.

\section{ADDITIONAL COMMENTS.}

\section{A. States localized near the boundary.}

We assumed throughout the paper that the center of an ALS is located far enough from the sample edge. For a quasi-1D sample, this means that $\xi_{\text {ef }} \ll L_{+}, L_{-}$. In the $2 \mathrm{D}$ case this implies that the distance from the observation point to the boundary is of the same order of magnitude in all directions, so that $\ln (L / l)$ is defined without ambiguity. Here, we will consider briefly the role of ALS situated close to the boundary, when these conditions are violated.

We start from the quasi-1D geometry. Let us calculate the distribution function $\mathcal{P}(u)$ in a point located very close to one of the sample edges. Formally, this means that $L_{-} \ll \xi_{e f}$. Then the function $W^{(1)}\left(u S \xi, \tau_{-}\right)$in eq.(3.4) can be approximated by unity, and we get

$$
\mathcal{P}(u)=\frac{2}{\pi} \xi^{3 / 4} S^{1 / 4} L^{-1 / 2} u^{-3 / 4} \exp \left\{-2 \sqrt{u \xi S}+\frac{\pi^{2} \xi}{4 L_{+}}\left(1-\frac{\sqrt{\xi / u S}}{L_{+}}+\ldots\right)\right\}
$$


We see therefore that close to the boundary the distribution $\mathcal{P}(\sqcap)$ has the asymptotic decay $\mathcal{P}(u) \sim \exp \{-2 \sqrt{u S \xi}\}$, which is slower than in the bulk of the sample, $\mathcal{P}(u) \sim$ $\exp \{-4 \sqrt{u S \xi}\}$. This means that if we consider the distribution $\mathcal{P}(u)$ averaged over the position of the observation point, its asymptotic tail will be always dominated by contribution of the points located close to the boundary, $\mathcal{P}(u) \sim \exp \{-2 \sqrt{u S \xi}\}$. This could be already

anticipated from eq.(3.46), where the factor $\exp \left\{\frac{\pi^{2}}{4}\left(\frac{\xi}{L_{+}}+\frac{\xi}{L_{-}}\right)\right\}$strongly increase with approaching one of the sample edges. The same tendency, but in a weaker form, is observed in eqs.(2.10), (2.11), (2.12). Calculating the average intensity $\left\langle\left|\psi^{2}(r)\right|\right\rangle_{u}$ of the corresponding ALS, we find that at $r>l$ eqs.(3.50), (3.54) retain their validity, with an additional overall factor of 2 . At small $r$, eq.(3.44) is slightly modified:

$$
\left\langle\left|\psi^{2}(r)\right|\right\rangle_{u}=\left(\frac{u}{\xi S}\right)^{1 / 2}\left[1+\sqrt{u S \xi} k_{d}(r)\right]
$$

In $2 \mathrm{D}$, we can consider a sample of the semicircular shape, with the observation point located in the center of the diameter serving as a boundary. The saddle-point solution then has exactly the same form (4.2), and the ALS intensity is still given by eq.(4.5), with an additional factor 2 . The asymptotic form of the distribution function $\mathcal{P}(u)$ gets an extra factor $1 / 2$ in the exponent:

$$
\mathcal{P}(u) \sim \exp \left\{-\frac{\pi^{2} \nu_{0} D}{2} \frac{\ln ^{2}\left(\frac{V u}{2 \pi^{2} \nu_{0} D} \ln \frac{L}{l_{*}}\right)}{\ln \left(L / l_{*}\right)}\right\}
$$

We expect this result to be applicable to any $2 \mathrm{D}$ sample of a characteristic size $L$, with a smooth boundary and the observation point taken in the vicinity of the boundary.

We see therefore, that, very generally, the probability of formation of an ALS with the center in a given point is strongly enhanced (via an extra factor $1 / 2$ in the exponent), if this point lies close to the sample edge. This leads to the additional factor $1 / 2$ in the exponent in the asymptotical form of the distributions $\mathcal{P}(u)$ and $\mathcal{P}(\rho)$ near the boundary.

\section{B. Orthogonal symmetry class.}

All the considerations in this paper can be straightforwardly generalized to the systems with unbroken time reversal invariance (orthogonal symmetry class). The main results are as follows:

i) all formulas for the average spatial density $\left\langle\left|\psi^{2}(r)\right|\right\rangle$ in the metallic samples retain their validity. In particular, in the quasi-1D case, eqs.(3.43), (3.44), (3.50), (3.54) hold with the same definition of $\xi=2 \pi \nu_{0} S D$. In the far localized tail, eq.(3.42), $\xi$ is replaced by $\xi / 2$, which is just the conventional dependence of the localization length on the symmetry of ensemble;

ii) in the expressions for the asymptotics of all distribution functions, an extra factor $1 / 2$ appears in the exponent;

iii) GUE-type fluctuations are replaced by the GOE-type ones, where appropriate. 


\section{SUMMARY.}

In this paper, we have studied the spatial structure of the anomalously localized sates in weakly disordered samples. Such states appear to govern the asymptotical behavior of the distribution function $\mathcal{P}(u)$ of local amplitudes of eigenfunctions. In the quasi-1D geometry, an ALS has an effective localization length $\xi_{\text {ef }}$ much shorter than the conventional one $\xi$. We were able to calculate exactly the average intensity of such a state. The found spatial distribution of the ALS intensity, $\left\langle\left|\psi^{2}(r)\right|\right\rangle \propto 1 /\left(r+\xi_{e f}\right)^{2}$, turned out to be in agreement with the form of the solution of the saddle-point equation of Ref. [21]. Thus, the saddle-point configuration indeed describes the average intensity of an ALS, as was conjectured in Refs. [22,21]. This allowed us to describe the spatial structure of ALS in 2D and 3D, as well. We have also studied the fluctuations of the ALS intensity and found them to be essentially of the same GUE type, as for a typical delocalized eigenstate.

Not only the asymptotic behavior of $\mathcal{P}(u)$, but also that of distributions of other quantities can be governed by a kind of ALS. Here are several important examples of such quantitites: inverse participation ratio $I_{2}$, relaxation time $t_{\phi}$, local density of states $\rho(E, r)$, global density of states $\nu(E)$. We have found that the spatial structure of ALS relevant to the asymptotic behavior of different distributions may be different. This is because an ALS constitutes an optimal fluctuation for one of the above quantities, and the form of this fluctuation depends on the specific characteristic, which is to be optimized. Finally, we have discussed interrelations between asymptotics of various distributions mentioned above. In the quasi-1D and 2D cases, this allowed us to present a comprehensive picture, which explains all the asymptotics as governed by exponentially rare events of formation of ALS.

\section{ACKNOWLEDGMENTS.}

The author is grateful to D.E.Khmelnitskii for discussions, and to Y.V.Fyodorov for discussions and critical reading of the manuscript. This work was supported by SFB 195 der Deutschen Forschungsgemeinschaft.

\section{APPENDIX. JOINT DISTRIBUTION FUNCTION OF EIGENFUNCTION INTENSITIES IN TWO DIFFERENT SPATIAL POINTS.}

In this Appendix, we write down the results for the joint distribution function of intensities of an eigenfunction in two spatial points,

$$
\mathcal{P}(\sqcap, \sqsubseteq ; \nabla)=\left\langle\delta\left(\left|\psi(\prime)^{\epsilon}\right|-\sqcap\right) \delta\left(\left|\psi(\nabla)^{\epsilon}\right|-\sqsubseteq\right)\right\rangle,
$$

for a quasi-1D system. For $l<r \ll \xi$, the function $\mathcal{P}(u, v)$ can be restored from its moments, eq.(3.61). The result is obtained in the following form:

$$
\begin{aligned}
\mathcal{P}(u, v ; r) & =\frac{1}{\pi^{2} V} \frac{\partial^{2}}{\partial u \partial v} \frac{1}{\sqrt{u v}} \int_{0}^{\infty} d \nu \nu \sinh (\pi \nu) e^{-\tau_{1}\left(1+\nu^{2}\right) / 4} \int_{0}^{1} d z \frac{1}{\sqrt{1-z}} W^{(1)}\left(\frac{v \xi}{1-z}, \tau_{2}\right) \\
& \times K_{i \nu}\left(2 \sqrt{\frac{v \xi}{1-z}}\right) \frac{\partial}{\partial z}\left\{\frac{1}{\sqrt{z}} W^{(1)}\left(\frac{u \xi}{z}, \tau_{-}\right) K_{i \nu}\left(2 \sqrt{\frac{u \xi}{z}}\right)\right\}
\end{aligned}
$$


In the opposite case, $r<l$, the expression (3.75) for the moments has to be used, yielding

$$
\begin{aligned}
\mathcal{P}(u, v ; r) & =\left.\frac{\xi}{L} \frac{\partial}{\partial u} \frac{\partial}{\partial v} \int_{0}^{2 \pi} \frac{d \phi}{2 \pi} \frac{\partial}{\partial z}\left\{W^{(1)}\left(z, \tau_{+}\right) W^{(1)}\left(z, \tau_{-}\right)\right\}\right|_{z=z(u, v, r, \phi)} \\
z(u, v, r, \phi) & =\xi S\left(\frac{u}{1+\sqrt{k_{d}(r)} e^{i \phi}}+\frac{v}{1+\sqrt{k_{d}(r)} e^{-i \phi}}\right)
\end{aligned}
$$

Unfortunately, even in the simplest case of an infinitely long sample, when the function $W^{(1)}$ is given by eq.(3.32), formulas (A.2) and (A.3) are too involved. This is why we have chosen in Sec.III to analyze the expressions for the moments, rather than the distribution function itself. 


\section{REFERENCES}

[1] Mesoscopic Phenomena in Solids, ed. by B.L.Altshuler, P.A.Lee and R.A.Webb (North Holland, Amsterdam , 1991).

[2] B.L.Altshuler, V.E.Kravtsov, I.V.Lerner, in Mesoscopic Phenomena in Solids, Ref. [1].

[3] A.A.Abrikosov, Solid State Commun., 37, 997 (1981).

[4] B.L Altshuler, V.N.Prigodin, Zh.Eksp.Teor.Fiz. 95, 348 (1989) [Sov.Phys.JETP 68, 198 (1989)].

[5] B.L.Altshuler, V.N.Prigodin, Pis'ma Zh. Eksp. Teor. Fiz. 47, 36 (1988) [JETP Lett. 47, 43 (1988)].

[6] V.E.Kravtsov, Habilitationsschrift, Heidelberg, 1992.

[7] U.Meirav, M.A.Kastner, and S.J.Wind, Phys. Rev. Lett. 65, 771 (1990); U.Sivan et al., Europhys. Lett. 25, 605 (1994).

[8] R.A.Jalabert, A.D.Stone, Y.Alhassid, Phys.Rev.Lett., 68, 3468 (1992).

[9] H.-J.Stöckmann, J.Stein, Phys.Rev.Lett. 64, 2215 (1990); S.Sridhar, Phys.Rev.Lett. 67, 785 (1991).

[10] A.Kudrolli, V.Kidambi, S.Sridhar, Phys. Rev. Lett. 75, 822 (1995).

[11] A.D.Mirlin, Y.V.Fyodorov, J.Phys.A: Math.Gen. 26, L551 (1993).

[12] A similar idea was used to study the statistics of LDOS and tunneling conductances, see Refs. [13 16].

[13] K.B.Efetov, V.N.Prigodin, Phys.Rev.Lett. 70 (1993), 1315.

[14] V.N.Prigodin, K.B.Efetov, S.Iida, Phys.Rev.Lett. 71, 1230 (1993).

[15] A.D.Mirlin, Y.V.Fyodorov, Phys.Rev.Lett. 72, 526 (1994); A.D.Mirlin, Y.V.Fyodorov, J.Phys. France I, 4, 665 (1994).

[16] A.D.Mirlin, Y.V.Fyodorov, Europhys. Lett. 25, 669 (1994).

[17] Y.V.Fyodorov, A.D.Mirlin, Phys.Rev.Lett. 67, 2405 (1991).

[18] Y.V.Fyodorov, A.D.Mirlin, Phys.Rev.Lett. 69, 1093 (1992).

[19] Y.V.Fyodorov, A.D.Mirlin, Int.J.Mod.Phys.B 8, 3795 (1994).

[20] Y.V.Fyodorov, A.D.Mirlin, Phys.Rev. B 51, 13403 (1995).

[21] V.I.Fal'ko, K.B.Efetov, preprints cond-mat/9503096, cond-mat/9507091.

[22] B.A.Muzykantskii, D.E.Khmelnitskii, Phys.Rev. B 51, 5480 (1995).

[23] A.D.Mirlin, Pis'ma Zh. Eksp. Teor. Fiz. 62, 583 (1995) [JETP Lett. 62, ... (1995)].

[24] A.D.Mirlin, Phys.Rev.B, to appear (15 January 1996).

[25] K.B.Efetov, Adv.Phys. 32, 53 (1983).

[26] J.J.M.Verbaarschot, H.A.Weidenmüller, M.R.Zirnbauer, Phys.Rep. 129, 367 (1985).

[27] M.L.Mehta, Random Matrices (Academic, New York, 1990).

[28] We are considering a quasi-1D sample, which means that its length $L$ is much larger than the transverse size $a$. However, $a$ is still supposed to be much larger than the wave length: $a k_{F} \gg 1$. On a scale shorter than $a$, the sample geometry is then not of $1 \mathrm{D}$, but rather of $2 \mathrm{D}$ or $3 \mathrm{D}$ nature, which is reflected in the Friedel function (3.21).

[29] H.Bateman, A.Erdélyi, Higher Transcendental Functions, vol.2 (McGraw Hill, New York, 1953).

[30] V.N.Prigodin, Phys.Rev.Lett. 74, 1566 (1995).

[31] Y.V.Fyodorov, A.D.Mirlin, Phys.Rev.Lett. 71, 412 (1993).

[32] Y.V.Fyodorov, A.D.Mirlin, unpublished. 
[33] B.A.Muzykantskii, D.E.Khmelnitskii, Pis'ma Zh. Eksp. Teor. Fiz. 62, 68 (1995) [JETP Lett. 62, 76 (1995)].

[34] B.Shapiro, Phys. Rev. Lett. 65, 1510 (1990). 